\title{
MicroRNAs and their isomiRs function cooperatively to target common biological pathways
}

Nicole Cloonan ${ }^{*}$, Shivangi Wani ${ }^{1}$, Qinying Xu¹, Jian Gu², Kristi Lea², Sheila Heater ${ }^{2}$, Catalin Barbacioru², Anita L Steptoe ${ }^{1}$, Hilary C Martin ${ }^{1}$, Ehsan Nourbakhsh ${ }^{1}$, Keerthana Krishnan ${ }^{1}$, Brooke Gardiner ${ }^{1}$, Xiaohui Wang ${ }^{3}$, Katia Nones ${ }^{1}$, Jason A Steen ${ }^{1}$, Nicholas A Matigian' ${ }^{1}$, David L Wood ${ }^{1}$, Karin S Kassahn ${ }^{1}$, Nic Waddell ${ }^{1}$, Jill Shepherd ${ }^{1}$, Clarence Lee ${ }^{4}$, Jeff Ichikawa ${ }^{4}$, Kevin McKernan ${ }^{4}$, Kelli Bramlett ${ }^{2}$, Scott Kuersten ${ }^{2,5^{*}}$ and Sean M Grimmond ${ }^{1 *}$

\begin{abstract}
Background: Variants of microRNAs (miRNAs), called isomiRs, are commonly reported in deep-sequencing studies; however, the functional significance of these variants remains controversial. Observational studies show that isomiR patterns are non-random, hinting that these molecules could be regulated and therefore functional, although no conclusive biological role has been demonstrated for these molecules.

Results: To assess the biological relevance of isomiRs, we have performed ultra-deep miRNA-seq on ten adult human tissues, and created an analysis pipeline called miRNA-MATE to align, annotate, and analyze miRNAs and their isomiRs. We find that isomiRs share sequence and expression characteristics with canonical miRNAs, and are generally strongly correlated with canonical miRNA expression. A large proportion of isomiRs potentially derive from AGO2 cleavage independent of Dicer. We isolated polyribosome-associated mRNA, captured the mRNAbound miRNAs, and found that isomiRs and canonical miRNAs are equally associated with translational machinery. Finally, we transfected cells with biotinylated RNA duplexes encoding isomiRs or their canonical counterparts and directly assayed their mRNA targets. These studies allow us to experimentally determine genome-wide mRNA targets, and these experiments showed substantial overlap in functional mRNA networks suppressed by both canonical miRNAs and their isomiRs.

Conclusions: Together, these results find isomiRs to be biologically relevant and functionally cooperative partners of canonical miRNAs that act coordinately to target pathways of functionally related genes. This work exposes the complexity of the miRNA-transcriptome, and helps explain a major miRNA paradox: how specific regulation of biological processes can occur when the specificity of miRNA targeting is mediated by only 6 to 11 nucleotides.
\end{abstract}

\section{Background}

MicroRNAs (miRNAs) are an important class of noncoding regulatory RNAs, which interfere with the translation of protein-coding mRNA transcripts. By incorporation into the RNA induced silencing complex (RISC), miRNAs can inhibit translation [1-6], promote sequestration of mRNAs to P-bodies [7], and/or

\footnotetext{
* Correspondence: n.cloonan@imb.uq.edu.au; Scott.Kuersten@epibio.com; s. grimmond@uq.edu.au

'Queensland Centre for Medical Genomics, Institute for Molecular Bioscience, The University of Queensland, St Lucia, Queensland, 4072, Australia

2 Life Technologies, 2130 Woodward St, Austin, TX 78744, USA

Full list of author information is available at the end of the article
}

destabilize and degrade target mRNAs [8-10]. Accumulating evidence suggests that miRNAs are required for achieving precise biological outcomes, such as developmental programs [11-14], and that dysregulation of miRNA expression can drive tumorigenesis and other human pathologies [15-17].

Due to their biological importance, intensive research has focused on understanding the biogenesis of miRNAs (reviewed in [18]). Precursor-miRNA hairpins (pre-miRNAs; approximately 60 to 110 nucleotides) are usually generated in one of two ways: either from the action of Drosha (an RNAse III enzyme) [19,20] on independent

\section{Biomed Central}


primary-miRNA (pri-miRNA) genes; or from Spliceosome-mediated processing of mRNA introns [21,22]. The pre-miRNAs are exported from the nucleus to the cytoplasm [23], where Dicer (another RNAse III enzyme) cleaves the hairpin to produce a miRNA duplex $[24,25]$. One strand of this duplex is incorporated into RISC $[24,26]$, becoming the mature miRNA (approximately 22 nucleotides). The other strand is often referred to as the miRNA* (miRNA-star), and is often thought to be non-functional and degraded [18,27], although some miRNA* products can be loaded into RISC [28-30]. Recently, an alternative biogenesis pathway was reported for the human miR-451, by which argonaut 2 (AGO2) cleaves the pre-miRNA to generate an intermediate molecule [31], the AGO2-cleaved premiRNA (ac-pre-miRNA). The ac-pre-miRNAs are processed to mature miRNAs by exonucleolytic trimming $[32,33]$. It remains to be seen what proportion of miRNAs are capable of processing via this alternative pathway.

The small size of mature miRNAs (typically only 20 to 24 nucleotides) makes them ideal for characterization using short-tag RNA-sequencing (RNA-seq) technologies [34,35]. Unlike hybridization approaches such as microarray profiling or Northern blotting, massive-scale sequencing provides a way to discriminate discrete but closely related RNA molecules, and profile miRNAs without a priori knowledge of expression [36]. RNA-seq has been used to study the miRNA content of a wide variety of species, tissues, and pathologies, with the striking and unexpected observation that pre-miRNAs almost always give rise to more than one mature miRNA sequence regardless of the sequencing platform used [37-46]. These miRNA variants have been dubbed 'isomiRs' [37], and can encompass substitutions, insertions or deletions, 3 ' end non-templated additions, and 5 ' and/or 3' cleavage variations.

Despite their consistent appearance in datasets, the biological relevance of isomiRs remains controversial. IsomiRs are commonly dismissed as sequencing artifacts [38,39], alignment artifacts [40], poor quality or degraded RNA [41], sloppy Drosha/Dicer excision $[42,43]$, or simply as 'trivial variants' [44], although some argue that measurement noise cannot account for the high frequency of these variants $[45,46]$. Recent analyses suggest that some isomiRs may be non-randomly distributed $[47,48]$, which hints that isomiR biogenesis could be regulated and therefore perhaps functional. These observational studies, however, do not suggest possible functions for isomiRs, and they are still potentially confounded by the high error rates of massivescale sequencing.

Although miRNA populations are thought to be relatively noncomplex (there are only 1,100 human miRNAs annotated in miRBase v15 [49]), the high level of error in massive-scale sequencing combined with the number of possible variants requires ultra-deep sequencing to ensure that isomiRs and poorly expressed miRNAs are detected reliably. Additionally, to understand the biological relevance of isomiRs, multiple biological states from multiple individuals need to be surveyed. In this study we seek to understand the biological relevance of isomiR expression by using ultra-deep miRNA-seq of ten adult human tissues from multiple individuals. We take a deliberately conservative approach to the detection of biologically relevant isomiRs, and validate their association with translating mRNAs. We also use biotinlabeled miRNAs and isomiRs to pull-down endogenous mRNA targets, finding that isomiRs act cooperatively with canonical miRNAs to target common biological pathways.

\section{Results}

Ultra-deep sequencing and alignment of miRNA-seq tags using miRNA-MATE

In order to thoroughly survey human miRNA expression, we constructed 30 libraries from 11 individual donors across 10 adult human tissues (brain, heart, kidney, liver, lung, ovary, placenta, spleen, testes, and thymus), and sequenced them using the SOLiD (Life Technologies) sequencing platform (Table 1; Table S1 in Additional file 1). As mature miRNAs are small, they contain limited information that can be used to place them uniquely in the genome (Figure S1 and Table S2 in Additional file 1). To maximize the accuracy of miRNA mapping, four parameters need to be optimized: (i) the appropriate choice of reference sequence; (ii) the number of mismatches allowed during alignment; (iii) the minimum length of tags to be aligned; and (iv) careful and conservative filtering of biological signal from noise.

To address these issues, we have created a miRNA-seq analysis pipeline ('miRNA-MATE'), which analyses SOLiD miRNA-seq data using two different alignment strategies (Figure S2 in Additional file 1). The first strategy, dubbed 'recursive mapping' [50], attempts to align sequences at their longest length, iteratively trimming and re-aligning if unsuccessful. This approach provides the best sensitivity for quantifying miRNA expression, but cannot determine the precise end of the captured miRNA. The second strategy ('adaptor trimming') was optimized for studying isomiRs, where knowing the exact length of the captured tag is crucial. For a full description of miRNA-MATE and mapping parameters used, see the Materials and methods, and Additional file 2.

In total, almost 430 million tags could be recursively aligned to miRNA hairpins represented in miRBase v15 
Table 1 Sequencing statistics for the small RNA tissue panel

\begin{tabular}{lcccrrrr}
\hline Tissue & $\begin{array}{c}\text { Number of } \\
\text { libraries }\end{array}$ & $\begin{array}{c}\text { Number } \\
\text { of runs }\end{array}$ & $\begin{array}{c}\text { Number of } \\
\text { individuals }\end{array}$ & $\begin{array}{r}\text { Mature mapped } \\
\text { tags (recursive) }\end{array}$ & $\begin{array}{r}\text { Mature mapped tags } \\
\text { (adaptor trimming) }\end{array}$ & $\begin{array}{r}\text { Hairpin mapped } \\
\text { tags (recursive) }\end{array}$ & $\begin{array}{r}\text { Hairpin mapped tags } \\
\text { (adaptor trimming) }\end{array}$ \\
\hline Brain & 1 & 2 & 1 & $3,967,251$ & $1,241,131$ & $2,582,668$ & $29,321,577$ \\
\hline Heart & 3 & 4 & 1 & $28,090,024$ & $3,306,067$ & $44,738,409$ & $38,556,827$ \\
\hline Kidney & 3 & 4 & 2 & $33,358,423$ & $34,126,218$ & $50,899,691$ & $23,032,850$ \\
\hline Liver & 3 & 4 & 2 & $20,138,397$ & $20,067,003$ & $30,613,223$ & $49,924,703$ \\
\hline Lung & 5 & 6 & 1 & $48,640,701$ & $45,419,062$ & $69,465,607$ & $46,059,416$ \\
\hline Ovary & 4 & 4 & 1 & $33,291,714$ & $34,240,510$ & $61,752,617$ & $32,597,796$ \\
\hline Placenta & 2 & 2 & 1 & $24,557,859$ & $19,582,092$ & $32,765,252$ \\
\hline Spleen & 3 & 4 & 1 & $28,616,021$ & $30,285,592$ & $42,144,297$ & $32,390,566$ \\
\hline Testes & 3 & 4 & 2 & $21,538,067$ & $22,458,615$ & $35,716,355$ & $28,385,304$ \\
\hline Thymus & 3 & 4 & 2 & $40,180,905$ & $39,883,625$ & $54,627,498$ & $43,278,516$ \\
\hline
\end{tabular}

[49], of which approximately $73 \%$ could also be precisely identified via the adaptor trimming approach (Table 1). To set a conservative threshold of genuine expression, we also aligned to an optimized reference library containing the mature miRNAs from all available species in miRBase v15 (Table 1). To examine cross-species mapping, we attempted to align all non-human, non-conserved miRNAs to human pre-miRNA hairpins without success (data not shown). Therefore, we would not expect short tags derived from a human library to align optimally to mature plant miRNAs unless they were generated by random error (for example, multiple templates on a single bead, contaminating signal from nearby beads or other causes). We could therefore use these non-human, non-conserved miRNAs to set a minimum threshold for genuine expression. In this set of libraries, miRNAs were considered as expressed if they were detected at $\geq 10$ transcripts per million (tpm) (Figure S3 in Additional file 1), and across all tissues we identified expression from 470 mature miRNAs from 423 hairpins (Table S3 in Additional file 1). Although this is a very conservative estimate of the noise level, this still allowed us to query the biological relevance of isomiRs with the open bias that highly expressed sequences are more likely to be biologically relevant. Hierarchical clustering of the miRNAs expressed above this threshold shows profiles clustering by tissue, confirming that the threshold was sufficiently conservative (Figure S4 in Additional file 1).

\section{Confirmation of sequencing data with quantitative RT- PCR and microarray analyses}

Quantitative RT-PCR (qRT-PCR) is often considered to be the gold standard for analysis of gene expression data. To confirm our sequencing results, and assess the accuracy and sensitivity of the different alignment strategies, we analyzed miRNA expression from the same RNA samples using TaqMan low density qRT-PCR arrays. Additionally, for one tissue (placenta) we also analyzed expression by Agilent miRNA microarray. These two validation strategies capture different attributes of the RNA sample. Agilent miRNA microarrays will capture signal from canonical miRNAs as well as isomiRs due to cross-hybridization with probes designed for canonical miRNAs. In contrast, TaqMan LDA qRTPCR is highly sequence-specific for canonical miRNAs. Figure 1 emphasizes this point, showing that the Spearman rank correlations for microarray and qRT-PCR data are best for recursive $(\rho=0.80)$ and canonical sequence-specific analyses $(\rho=0.76$; Figures S5 and S6 in Additional file 1), respectively. Both microarray and qRT-PCR show high concordance with the sequencing data, demonstrating our analysis is both sensitive and specific.

\section{A standard nomenclature for isomiR description}

Although the existing 'miRNA/miRNA-star' annotations give instant functional context, the major disadvantage when used as a nomenclature is that these annotations are not based on static characteristics of the miRNA the dominant strand could change in different biological settings leading to different names describing the same molecule. To investigate the scope of this problem, we examined how often the dominant arm of the premiRNA hairpin switched across different tissues. Of 163 hairpins with expression of $\geq 10 \mathrm{tpm}$ in both the $5 \mathrm{p}$ and $3 p$ arms, we identified 21 (12.9\%) whose dominant expression switched from one arm to the other in at least one tissue (Figure 2; Table S4 in Additional file 1). Considering that the tissues sampled here represent only a small fraction of known biological diversity, this figure represents a lower rather than upper bound for arm-switching. In this context, the star-based nomenclature is not-informative, and we (along with miRBase curators) argue that this nomenclature should be abandoned in favor of the ' $5 \mathrm{p}$ ' and ' $3 \mathrm{p}$ ' annotations. 


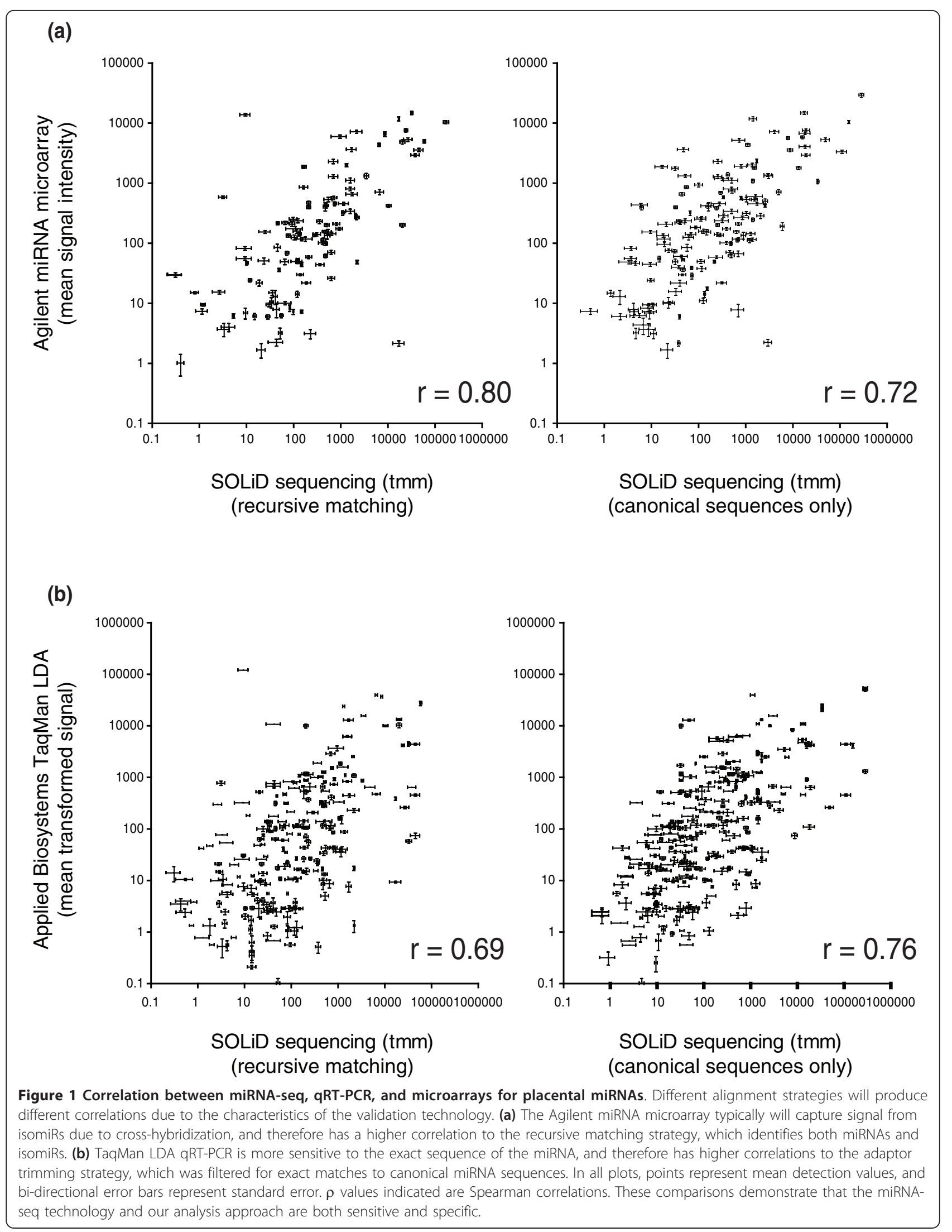




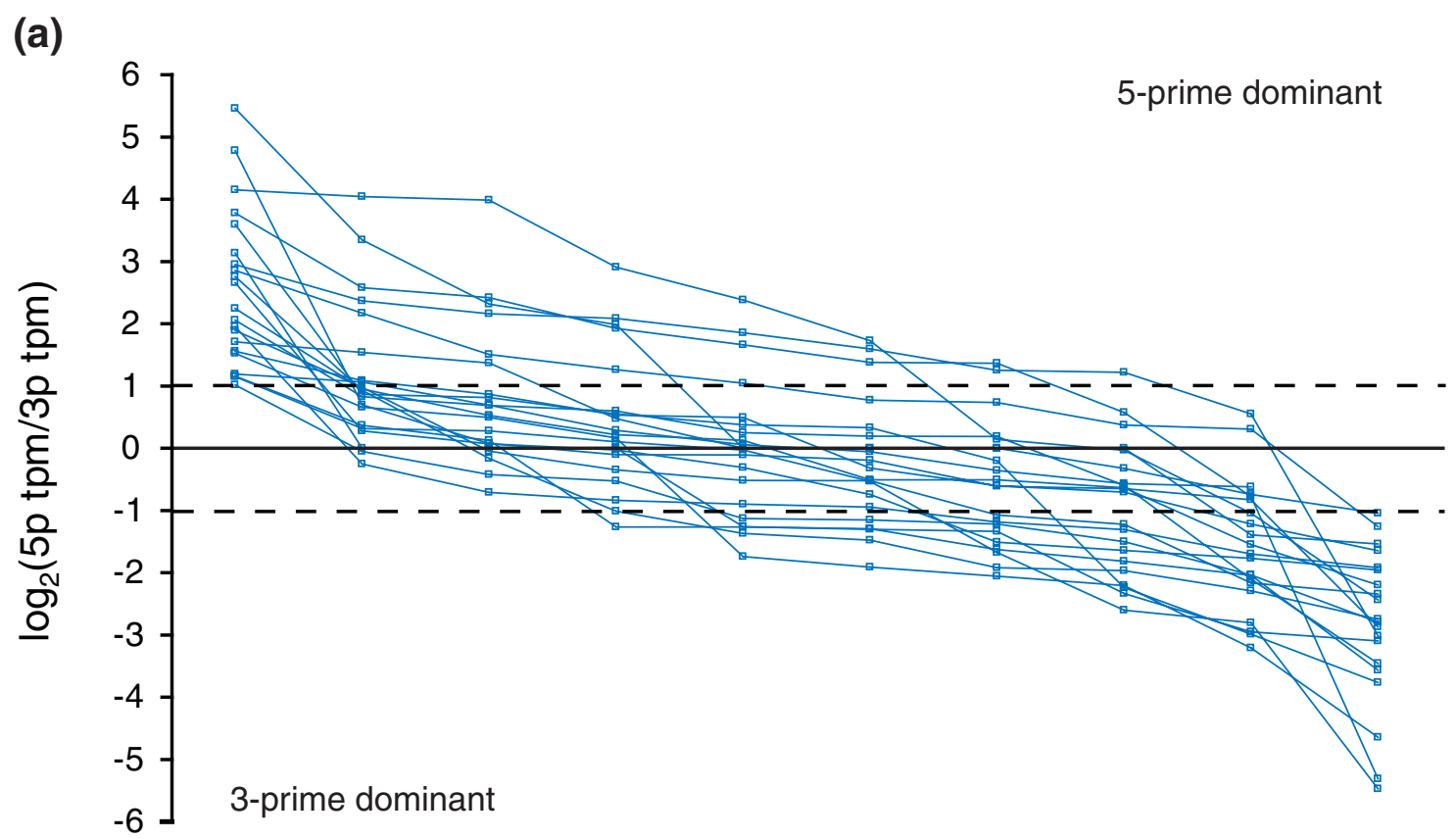

(b)

hsa-miR-126

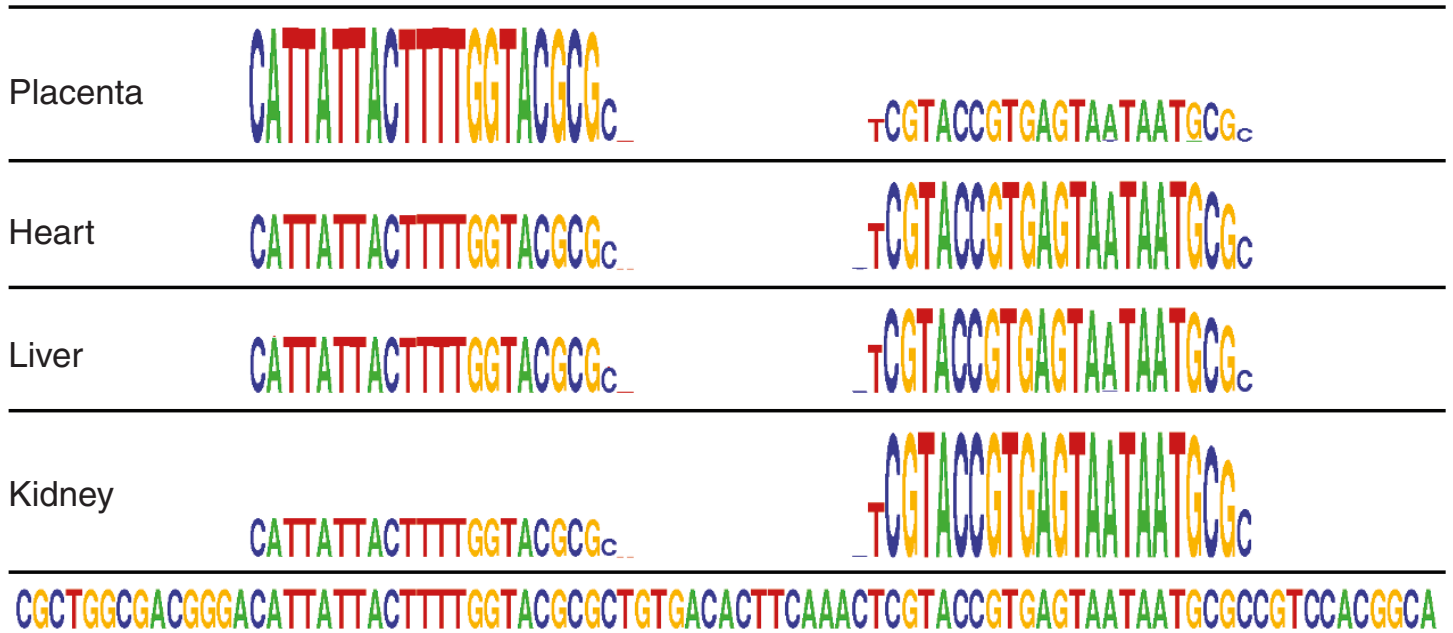

Figure 2 Arm switching frequency in the miRNA-seq data. (a) Arm-specific expression of at least 21 miRNAs varied between tissues. For all 10 tissues (points), log ratios of $5 p$ to $3 p$ arm expression for 21 miRNAs (blue lines) are plotted according to the arm on which dominant expression occurred, clearly illustrating differences between the tissues for each miRNA. (b) Sequence logo plots for hsa-miR-126 showing the switch between expression dominance from the $5 p$ arm (placenta) to the $3 p$ arm (heart, liver, and kidney). The pre-miRNA hairpin is displayed along the bottom.

We propose a machine parsable nomenclature based on the sequence of the isomiR and the relative positions in the pre-miRNA hairpin. The details are presented in Additional file 3, but as an example: the notation 'hsa-
miR-143-3p|\{hsa-miR-143\}|60_80|' refers to the canonical sequence of hsa-miR-143-3p (annotated in miRBase as hsa-miR-143); and 'hsa-miR-143-3p|\{isomiR\}|62_80| sub.77.G > A' refers to an isomiR of hsa-miR-143 that 
starts two nucleotides downstream of the canonical sequence, and contains a substitution from $\mathrm{G}$ to $\mathrm{A}$ at position 77 in the hairpin. Much of this nomenclature has been based on existing strategies for the description of mutations in nucleotide data, and should therefore be reasonably intuitive in its application to miRNAs. We have used this nomenclature for the remainder of this study. A tab delimited text file containing all detected isomiRs and their counts in each sample can be found in Additional file 4.

\section{Classification and characterization of isomiRs}

Having annotated our ultra-deep sequencing data with isomiR-specific nomenclature, we then sought to understand some of the basic characteristics of isomiRs and compared them to the characteristics of canonical miRNAs. We first classified the tags into seven mutually exclusive categories: (i) canonical miRNAs; (ii) start-siteonly isomiRs; (iii) end-site-only isomiRs; (iv) substitution-only isomiRs; (v) shifted isomiRs (those isomiRs where the length is identical to the canonical sequence, but there are variations in both the start and end sites); (vi) 3' non-templated addition isomiRs (isomiRs where additional nucleotides are present at the 3' end that do not match the reference hairpin sequence); and (vii) the remaining 'mixed' isomiRs, which are typically combinations of the prior categories (Figure 3a). To ensure the robustness of our results, we required that an isomiR be present at a minimum of $10 \mathrm{tpm}$ in every sample of at least one tissue, and that the isomiR must have been detected by at least two different library preparation methods. This effectively excluded isomiRs that were present only in the brain and placenta, and isomiRs that were specific to an individual donor (Table S1 in Additional file 1). We also excluded 'orphan' isomiRs (isomiRs for which the canonical miRNA sequence was not detected (Table S5 in Additional file 1)), as these could possibly arise from mis-annotated mature miRNAs. Applying these additional filters, we detected 190 canonical miRNAs and 834 isomiRs. As we did not detect any 3' non-templated addition isomiRs above the expression threshold in any tissue, this class of miRNA was not considered further in this study.

We then sought to examine the expression characteristics across each of these categories (Figure $3 \mathrm{~b}$ ). In most tissues, start-site isomiRs, substitution isomiRs, and mixed isomiRs show greatly reduced expression compared to the canonical miRNAs, whereas end-site isomiRs and shifted isomiRs both show either comparable or slightly reduced expression. We hypothesized that the limited expression of substitution-only isomiRs was predominantly due to sequencing errors, and that the expression ratios of these isomiRs would be very low compared to the corresponding canonical miRNA.
Typically, the median ratio for substitution-only isomiR tpm/canonical miRNA tpm was around $1 / 1,000$, and there were very few instances of this ratio exceeding $1 /$ 10 , suggesting that this category cannot be distinguished from sequencing artifacts (Figure 3c). In contrast, the median ratio for end-site-only isomiRs was approximately $1 / 3$, and there were a great many with ratios exceeding 1 , indicating that these isomiRs were more abundant than their canonical miRNAs. Start-site-only and shifted isomiRs also have a greatly increased median compared to substitution-only isomiRs: approximately $1 /$ 30 and approximately $1 / 10$, respectively. Together, these results suggest that most categories of isomiRs are unlikely to be merely artifacts of sequencing or library generation.

One isomiR feature that has been identified in previous studies but not confirmed here was the over-abundance of $U$ insertions when compared to $U$ deletions [51]. We find no substantial evidence for widespread insertion or deletion events in any tissue, identifying at most $0.54 \%$ of input tags as containing deletions, and $0.066 \%$ of tags containing insertions (Figure S7 in Additional file 1). This is likely due to the sequencing platforms used: in the Reid et al. study [51] the authors utilize 454 and Illumina sequencing, both of which are more susceptible to insertion or deletion artifacts than SOLiD sequencing used here. Such biases in miRNA detection have been seen previously [52], and highlight that the exact protocols to capture and measure isomiRs will influence the results, and should be considered when interpreting the relative importance of either individual isomiR sequences, or broad isomiR classes.

\section{The ac-pre-miRNA pathway contributes substantially to end-site only isomiRs}

The recent discovery of an alternative miRNA biogenesis pathway potentially explains the presence of a large proportion of the isomiR population. AGO2-mediated cleavage of the pre-miRNA produces intermediates (acpre-miRNA) that are detectable by miRNA-seq. We examined the sequence logos from the hsa-miR-451 pre-miRNA, known to participate in this pathway $[32,33]$, observing three major characteristics: (i) the consistency of the $5^{\prime}$ end of the mature sequence; (ii) the dominance of the 5' arm (while the 3' arm can sometimes be detected, it is not observed above $10 \mathrm{tpm}$ in any tissue); and (iii) the substantial tail of ac-premiRNA intermediates (Figure 4a). To understand the scope of ac-pre-miRNAs within the isomiR population, we searched for hairpins displaying these expression characteristics on either arm of the hairpin (Figure 4b), restricting our analysis to the eight tissues with multiple library preparation types (heart, kidney, liver, lung, ovary, spleen, testes, and thymus). In this way, we 
(a)

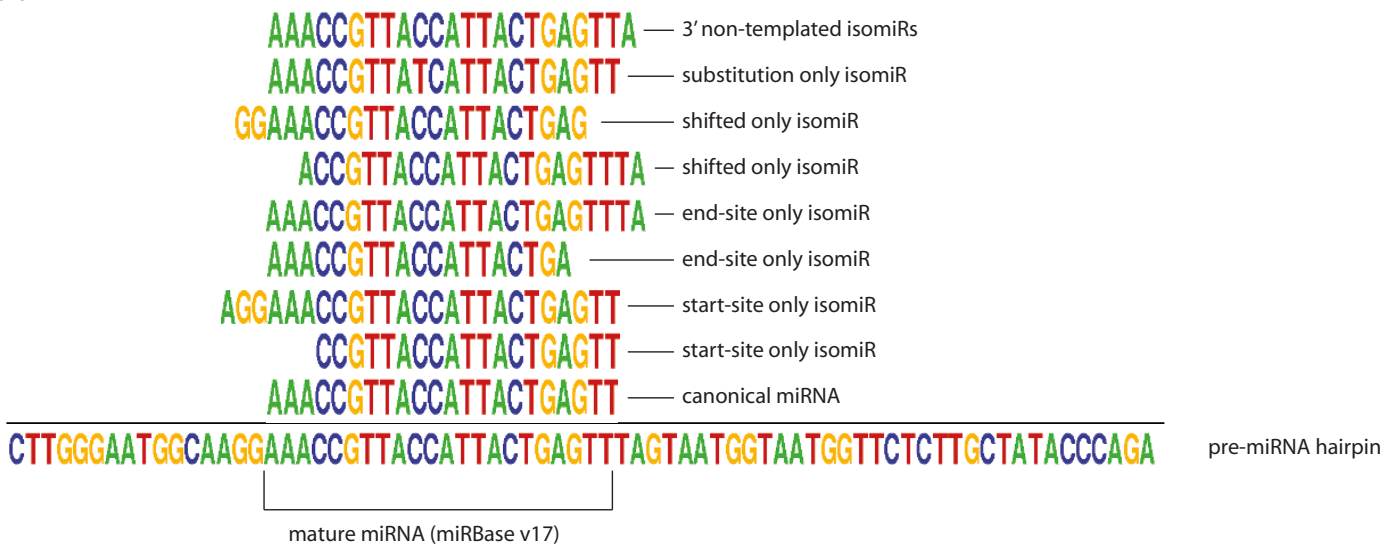

(b)

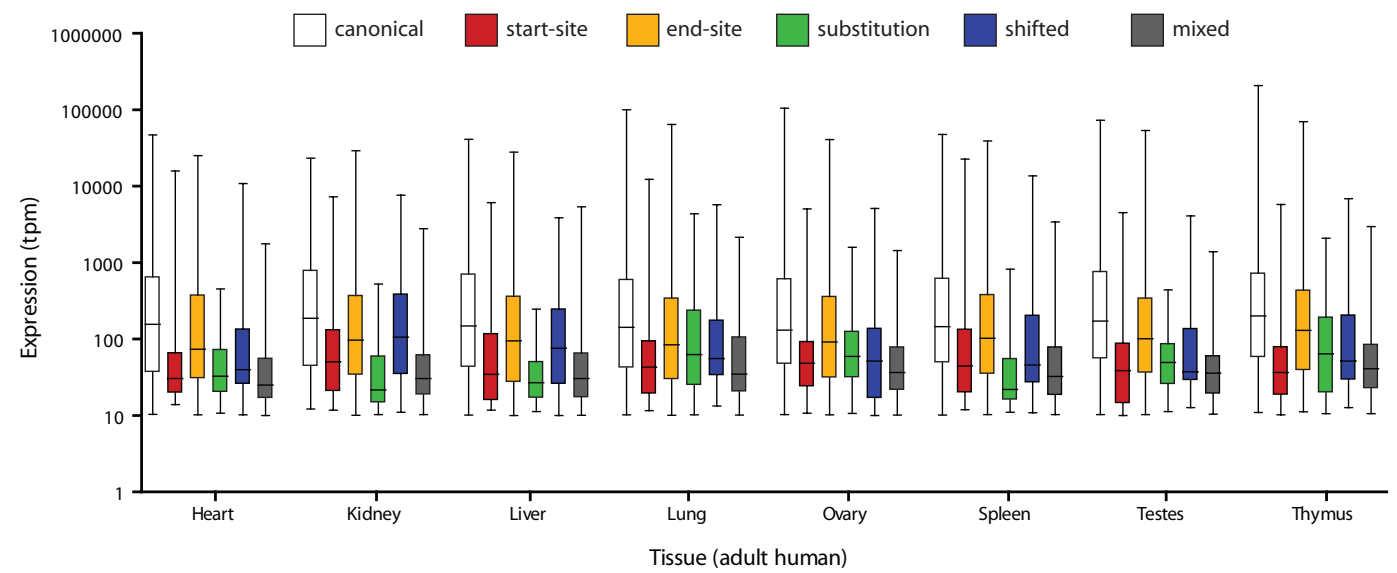

(c)

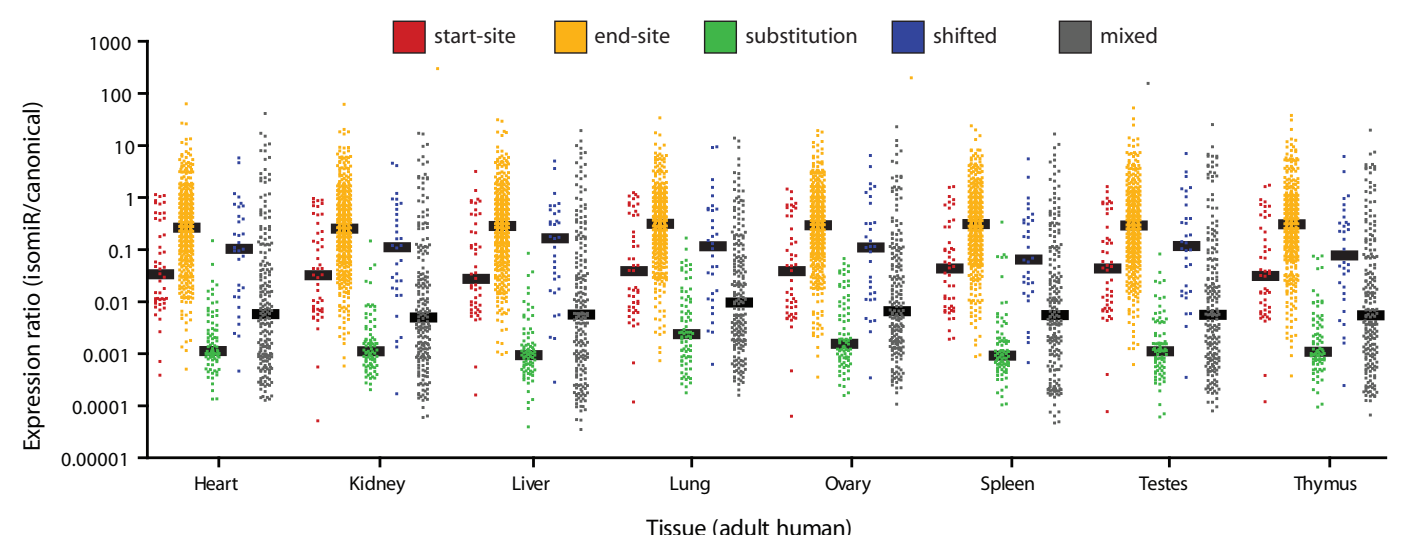

Figure 3 miRNA and isomiR characteristics. (a) Example of a canonical miRNA and each mutually exclusive isomiR category. IsomiRs that would fit more than one of these categories are classified as 'mixed' isomiRs. (b) Box-whisker plots showing expression of isomiR types within each tissue. (c) Ratio of each isomiR to its canonical miRNA grouped according to isomiR type within each tissue. 
(a)

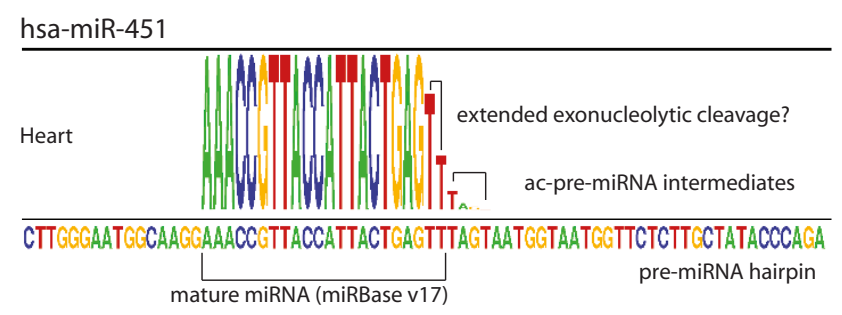

(c)

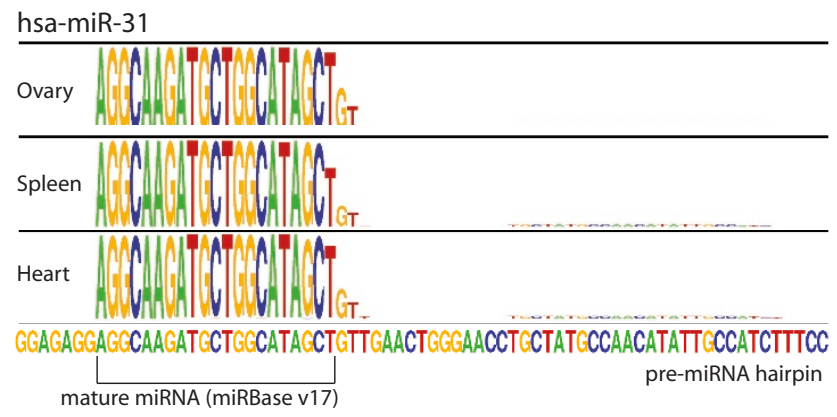

(d)

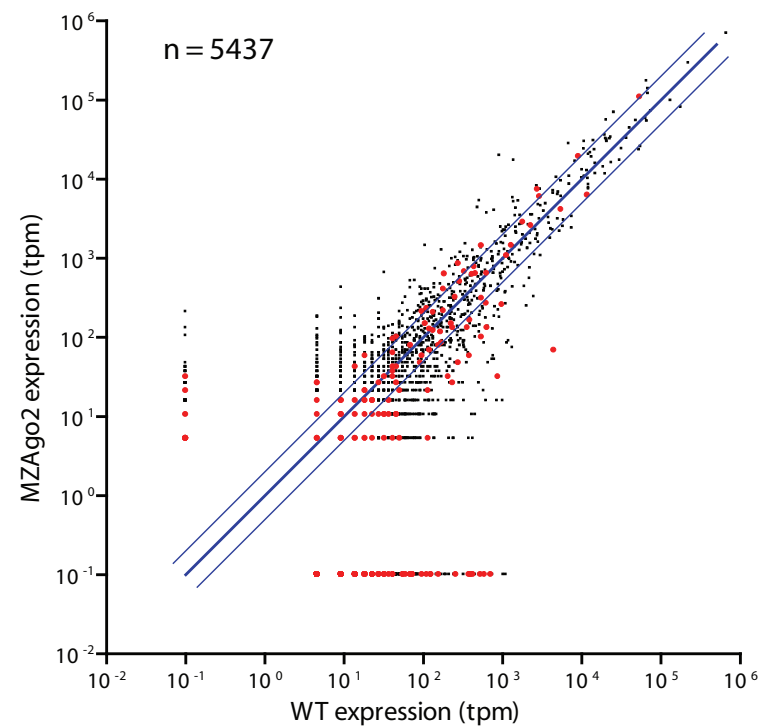

(b)

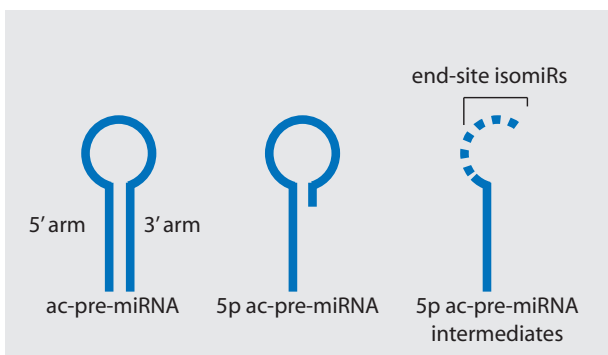

start-site isomiRs

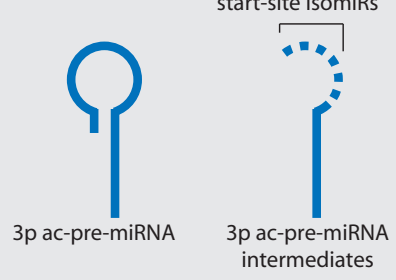

(e)

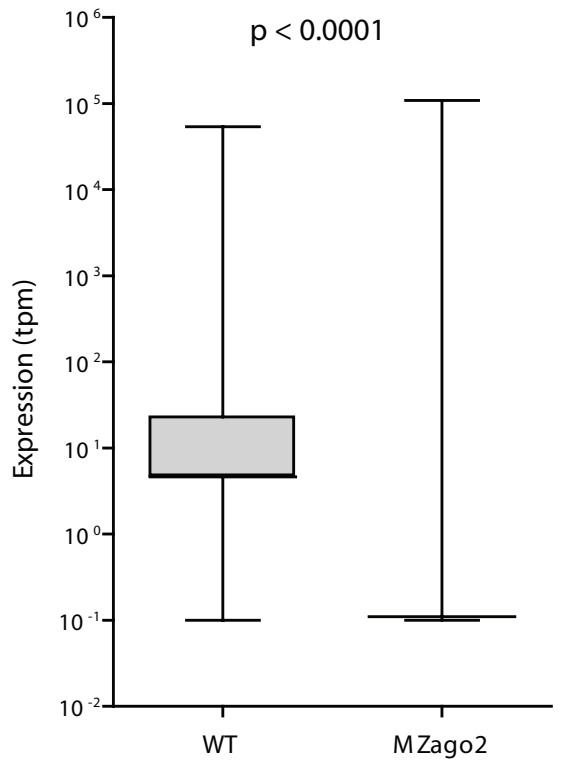

Figure 4 Identification of ac-pre-miRNA candidates. (a) Sequence logo profile of hsa-miR-451 showing $5^{\prime}$ arm dominance, almost no variation in the $3^{\prime}$ end of the mature miRNA, and a trail of $3^{\prime}$ isomiR extensions. (b) Schematic diagram illustrating the generation of end-site ( $5 p$-ac-pre-miRNA) and start-site (3p-ac-pre-miRNA) isomiRs via AGO2-mediated cleavage of a pre-miRNA. Dashed lines indicate variable regions. (c) Sequence logo profile for hsa-miR-31, which was identified by our analysis as a candidate for biogenesis through the ac-pre-miRNA pathway. Three representative tissues are shown. (d) Wild-type (WT) zebrafish whole embryo versus mutant AGO2 inactive (MZAgo2) whole embryo miRNA and isomiR expression. Each point represents a separate isomiR or miRNA. The thick blue line is the perfect correlation line, the thin blue lines are \pm 2 -fold visual guides. Red dots indicate isomiRs/miRNAs derived from candidate ac-pre-miRNA hairpins. A bias towards downregulation in AGO2 mutants is evident in this plot. (e) Box and whiskers plots showing the expression of all isomiRs/miRNAs deriving from acpre-miRNA candidates in wild-type (WT) and mutant (MZago2) miRNA-seq samples; 75\% of ac-pre-miRNA candidate molecules are not expressed in the mutant sample. 
identified an additional 46 5p-ac-pre-miRNA candidates (Figure 4c; Table S6 in Additional file 1), but did not find evidence of 3p-ac-pre-miRNAs, perhaps reflecting the relative activity of $5^{\prime}$ to 3 ' RNA exonucleases compared to 3' to 5' exonucleases [53]. Together, these potential ac-pre-miRNA intermediates account for $62.8 \%$ of the end-site isomiR expression, and $48.3 \%$ of all isomiR expression observed in this study.

To confirm the association of these ac-pre-miRNA candidates with AGO2-mediated cleavage, we examined the miRNA-seq profiles of wild-type zebrafish whole embryos, and mutant embryos where AGO2 was catalytically inactive [33]. Using miRNA-MATE to align these data to Danio rerio hairpins (miRBase v17), we identified 13 ac-pre-miRNA candidates with expression of the 5p canonical miRNA in the wild-type sample. As a group, the miRNAs and isomiRs deriving from the ac-premiRNA candidate hairpins have significantly lower expression in the AGO2 mutants (Figure 4d, e; Chisquare value $=12021.776 ; 1$ degree of freedom; $P<$ $0.0001)$. Of the 13 candidates, 9 had complete ablation of canonical expression with inactive AGO2, and an additional 3 had a reduction in expression of $>80 \%$. IsomiR expression patterns also support all but one of these 12 miRNAs, with expression $<80 \%$ of wild-type levels, leaving $11 / 13(84.6 \%)$ candidates with strong experimental support for AGO2 cleavage (Table S6 in Additional file 1). These data support a more extensive role for AGO2-mediated cleavage in miRNA/isomiR biogenesis than has previously been appreciated.

\section{IsomiRs are actively associated with the transcriptional machinery}

Previous studies have identified isomiRs associated with the AGO proteins $[45,54,55]$, suggesting that these molecules can incorporate into endogenous RISC. We sought to further clarify the biological relevance of isomiRs by determining whether these isomiR-containing RISC complexes were capable of binding to endogenous mRNAs. As the RISC complex is associated with translating mRNA (polysomes) [4,56], we purified polysomal RNA from HeLa cells using a sucrose gradient fractionation (Figure S8A in Additional file 1) followed by a standard column and ethanol concentration, designed to capture RNAs longer than 200 nucleotides, thus discarding any miRNAs not bound to long polysomal mRNA (Figure 5a). We then eluted the bound miRNAs using a gentle heating step, and prepared this miRNA fraction for sequencing (Figure 5a). We also prepared total cell miRNAs, and total cell RNA-bound miRNAs.

The quality of all RNA preparations was checked, revealing no evidence of degradation (Figure S8B in Additional file 1). We confirmed that the RNeasy columns did not retain miRNAs in the absence of longer
mRNAs by qRT-PCR of HeLa RNA processed in five ways: (1) RNeasy column (to confirm detection of miRNAs); (2) RNeasy column-purified RNA that has been heat denatured and re-purified on an RNeasy column (to remove the bound miRNAs); (3) RNeasy columnpurified RNA that has been re-purified on an RNeasy column (to confirm that miRNAs are stable after multiple purifications); (4) miRNeasy column (to confirm detection of miRNAs); and (5) miRNeasy column-purified RNA that has been heat denatured and re-purified on a miRNeasy column (to confirm that miRNAs can still be detected after heat denaturation). The selective loss of miRNA detection after heat denaturation and RNeasy purification, but not heat denaturation and miRNeasy purification, or double RNeasy purification confirms that miRNAs are stably associated with longer RNAs, and are not retained on the RNeasy column in the absence of longer RNAs (Figure S8C in Additional file 1). We also checked for the enrichment of polysomes in our sample preparations by assessing the miRNA-seq libraries for: (i) ribosomal RNA (expected to be enriched in the polysome fraction relative to mRNA associated fraction); (ii) tRNA (expected to be moderately depleted in the polysome fraction relative to the total RNA fraction); and (iii) small nucleolar RNA (snoRNA; expected to be greatly depleted in the polysome fraction). We found a 3.9-fold enrichment of rRNAs, an 8.1-fold depletion of tRNAs, and > 50-fold depletion of snoRNAs in polysome RNAs longer than 200 nucleotides when compared to total RNAs longer than 200 nucleotides. These data confirm that the polysome fraction is of sufficient purity to analyze miRNAs associated with actively translating ribosomes.

As expected, the expression levels of polysome-associated miRNAs were well correlated (Spearman $\rho=$ 0.62 ) with those of total mRNA-bound miRNAs (Figure $5 \mathrm{~b}$ ), confirming the dominant function for cytoplasmic RNA-bound miRNAs is to bind to mRNAs. The correlation between polysome miRNAs and total HeLa miRNAs was far less (Spearman $\rho=0.46$; Figure 5c), suggesting that there are a number of miRNAs that are not localized to the cytoplasm, likely reflecting the other non-targeting functions of miRNA [57]. The repertoire of isomiRs identified in the polysome miRNA fraction was similar to that found in the total RNA-bound fraction. Approximately $97 \%$ of isomiRs detected in the total RNA-bound fraction were also detected in the polysome fraction, and despite a polysome enrichment of at least four-fold, the distribution of isomiRs in these two fractions was essentially identical (Figure $5 \mathrm{~d}$, e). This demonstrates that isomiRs of all categories are associated with the translational machinery, and thus are highly likely to be functional in mammalian cells. We also compared the proportion of isomiRs from the 


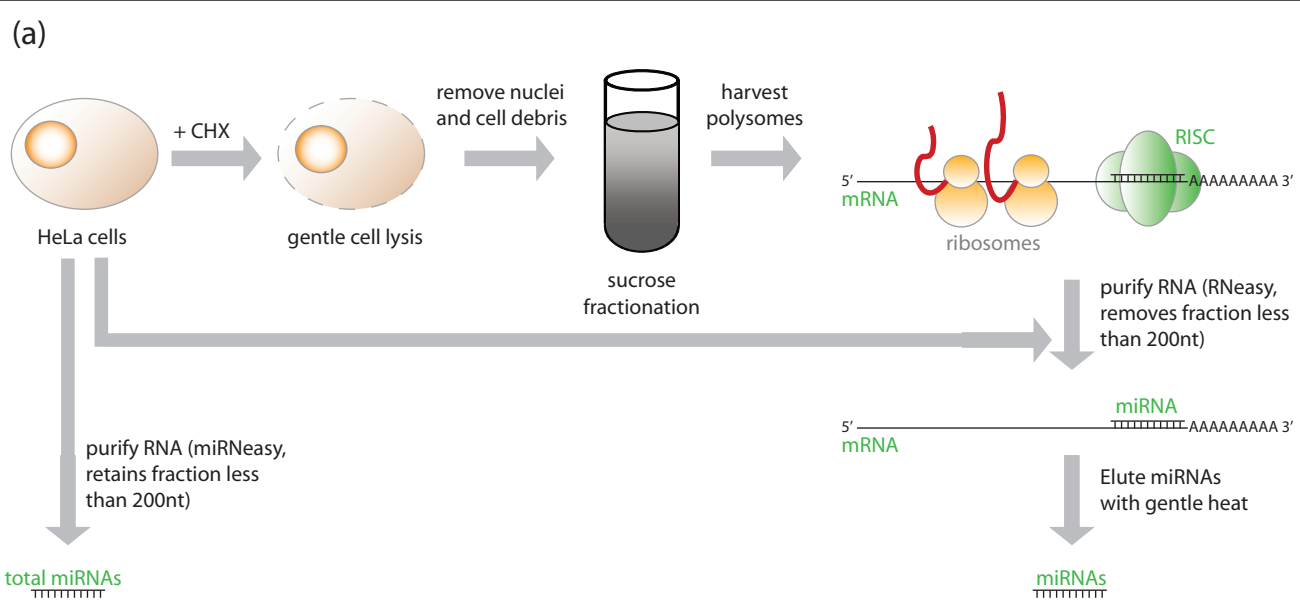

(b)

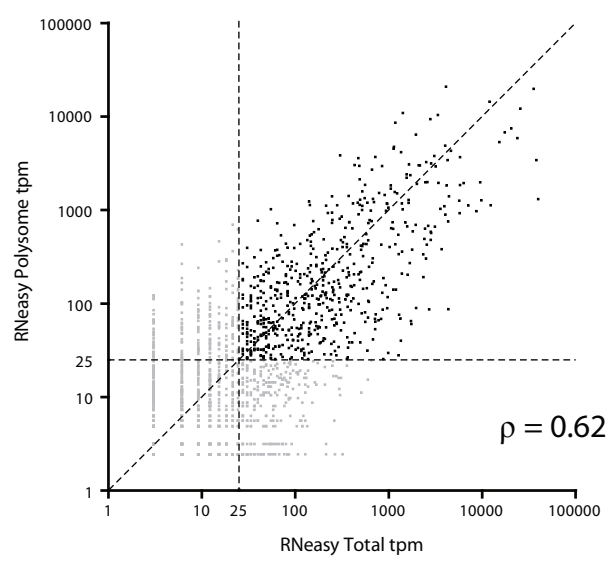

(d)

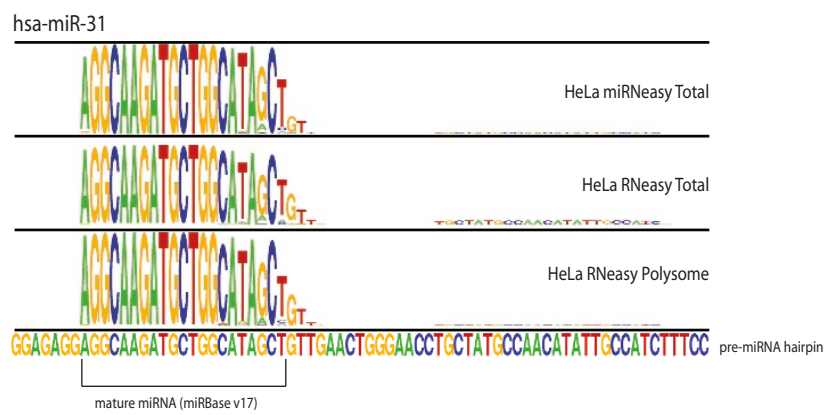

(c)

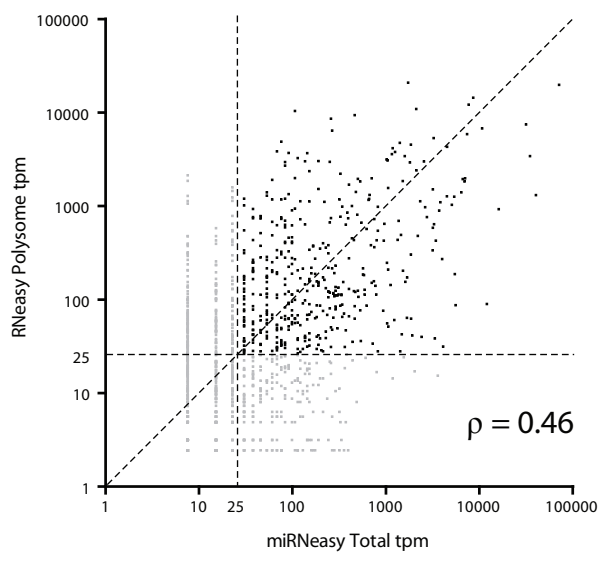

(e)

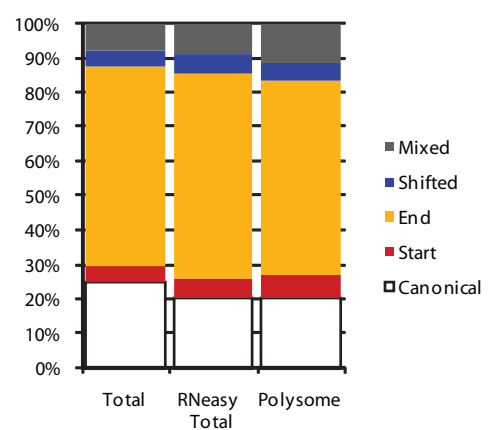

Figure 5 IsomiRs are associated with translational machinery. (a) Schematic diagram showing the laboratory workflow to isolate total miRNA, miRNA associated with mRNA, and miRNAs associated with polysomal mRNA. (b, c) Correlation scatter plots describing miRNA expression in the three preparations described in (a). The noise threshold was set at $25 \mathrm{tpm}$ for these libraries. (b) Correlation scatter plot between miRNA associated with mRNA (x-axis), and miRNA associated with polysomal mRNA (y-axis). The dashed diagonal line represents perfect correlation, while the horizontal and vertical lines represent the likely noise thresholds for these data. (c) Correlation scatter plot between total cellular miRNAs, and polysomal associated miRNA. The dashed diagonal line represents perfect correlation, while the horizontal and vertical lines represent the likely noise thresholds for these data. (d) A sequence logo profile for hsa-miR-31 showing similar profiles for all three HeLa miRNA-seq samples. This example shows an ac-pre-miRNA candidate hairpin with no change in the proportion of intermediates in the translational machinery. (e) Proportional bar graph illustrating the similar distribution of isomiR categories in each of the three HeLa miRNA-seq samples. CHX, cycloheximide, nt, nucleotide. 
ten adult human tissues, and checked for their expression in the HeLa polysome-associated fraction. Except for substitution only, and mixed isomiRs (which contain a large proportion of nucleotide changes), approximately $30 \%$ of the tissue atlas isomiRs in each category were also detected in the HeLa polysome fraction (Table S7 in Additional file 1), again confirming the relevance of these particular isomiR types.

\section{IsomiR expression is highly correlated with canonical miRNA expression}

Having determined that isomiRs are expressed at biologically meaningful levels, and that they are associated with the translational machinery, we then investigated the biological role of isomiRs and their relationship to canonical miRNAs. We hypothesized that if isomiRs were highly correlated with canonical miRNAs, they would be likely to drive similar biology. Equally, if they were not highly correlated, they would drive different biology. Across all categories of isomiRs, we found isomiRs to be highly correlated with canonical miRNA expression (Figure S9 in Additional file 1). Only small differences were observed between categories, with the median Pearson correlation ranging from approximately 0.9 for shifted isomiRs to approximately 0.96 for endsite isomiRs. In addition, more than $75 \%$ of isomiRs had Pearson correlations of $>0.75$ with their canonical miRNAs. Whilst we acknowledge that the small differences observed could potentially represent differences in isomiR biogenesis and regulation, we conclude that most isomiRs are likely to be driving similar biology to their canonical miRNAs.

\section{IsomiRs increase the specificity of miRNA targeting}

For miRNAs involved in the translational machinery, their biological role can be understood by the mRNA targets they repress. Target site prediction is generally based on 'seed' site interactions, although miRNAs can bind their targets in many different ways [58]. Additionally, existing prediction algorithms are notorious for false positives [59-61]. To determine the direct mRNA targets of miRNAs and isomiRs in a high-throughput manner, we used biotin-labeled synthetic miRNAs or isomiRs to pull-down endogenous mRNA targets and profiled these fractions by microarray (Figure 6a) $[62,63]$. We first optimized this technique for miR-17$5 \mathrm{p}$, a miRNA we have previously characterized as a regulator of cell cycle progression [64], and a miRNA for which there are more than 30 mRNA targets validated by reporter gene assay [64-67]. Using a threshold equivalent to a false discovery rate of $5 \%$, we were able to identify 21 of the 34 validated mRNA targets above this threshold (Figure S10A in Additional file 1). Additionally, gene set enrichment analysis (GSEA) of the
miR-17-5p pull-down genes corroborates the understood biology of this miRNA (Figure S10B in Additional file 1 ), confirming the sensitivity and specificity of this assay.

We then selected two closely related miRNAs (hsamiR-10a-5p|\{hsa-miR-10a\}|21_43| and hsa-miR-10b-5p| \{hsa-miR-10b\}|26_48|), and a shifted isomiR for each of these (hsa-miR-10a-5p|\{isomiR\}|22_44| and hsa-miR$10 \mathrm{~b}-5 \mathrm{p} \mid\{$ isomiR\}|27_49|), that displayed concordant expression across tissues (Figure 6b). We performed biotin pull-downs for each of these four molecules, independently replicated, and assayed the captured mRNAs by microarray. As expected by target prediction, each pull-down enriched expression of hundreds of mRNAs relative to mock transfection controls. Microarray results showed that between 492 and 1,208 genes were significantly enriched in the pull-downs, many of which were common to several of the four pull-downs (Figure 6c).

The miR-10 miRNAs are often dys-regulated in cancers, and have been shown to act as oncogenes by targeting the HOX genes and other tumor suppressors (reviewed in [68]). In our data set, we found that targets of miR-10 miRNAs and isomiRs were enriched in pathways that are directly involved in cancer (such as cell cycle regulation and apoptosis signaling), and in signaling pathways that contain important cancer molecules. One such pathway, 'the molecular mechanisms of cancer', was the most significant pathway for hsa-miR-10b5p|\{hsa-miR-10b $\}\left|26 \_48\right|\left(P=8.32 \times 10^{-7}\right)$, but was also significant for the other three pull-downs $(P=2.85 \times$ $10^{-3}$ to $9.33 \times 10^{-5}$ ). Many key genes of this pathway were targeted by multiple miRNAs/isomiRs (including those encoding RAS, PI3K, JNK, E2F, PKC, and HIPK2), but the combination of miRNAs/isomiRs varied from gene to gene (Figure 7). Such a targeting pattern suggests that isomiRs increase the dosage of miRNAmRNA targeting to core biological processes, whilst distributing the 'off-target' effects randomly through the transcriptome.

If hypothesis is true, it would follow that mRNAs targeted by multiple miRNAs/isomiRs would be enriched in functional networks compared to other parts of the transcriptome. To test this, we compared the repertoire of significantly enriched pathways identified when filtering the gene lists by the number of miRNAs/isomiRs that target these genes. Of the 44 significantly enriched pathways $(P \leq 0.01)$ identified by at least two pull-down experiments, we were able to identify 25 (56.8\%) and 29 (65.9\%) pathways when filtering for genes identified by at least two or three pull-downs, respectively. The converse also holds true - we collected all pull-down genes in a given pathway, where that pathway was significantly enriched $(P \leq 0.01)$ for at least one member of the miR- 


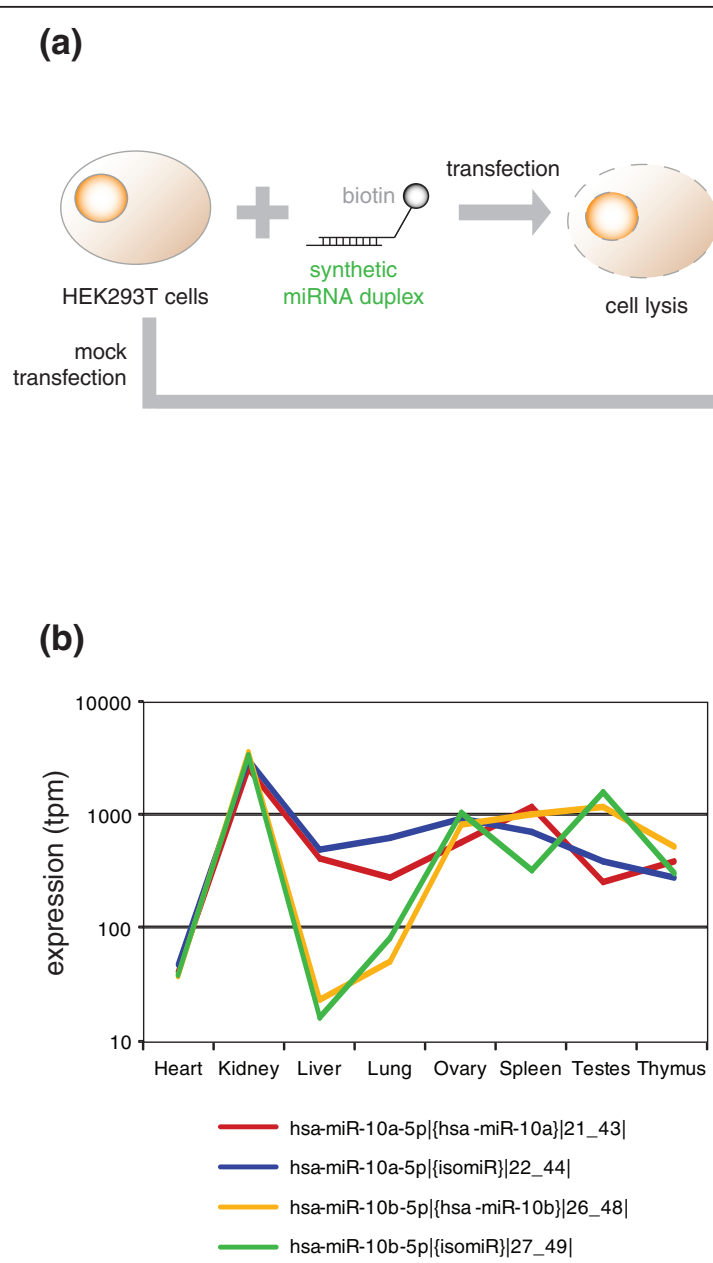

(d)

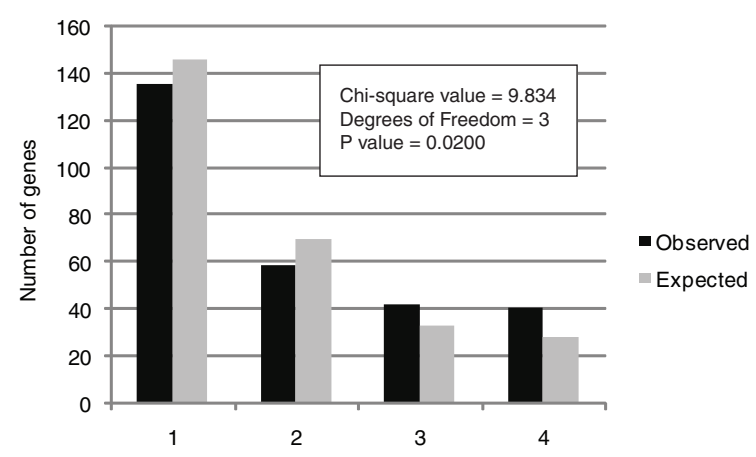

Number of miRNAs/isomiRs targeting a gene

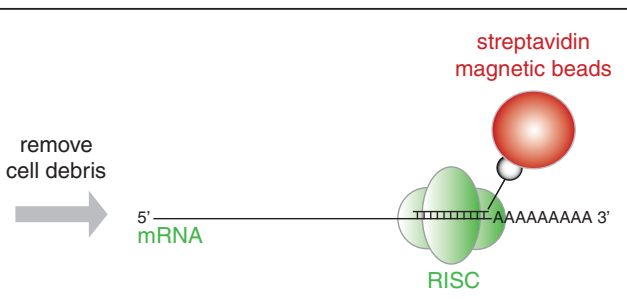

purify RNA

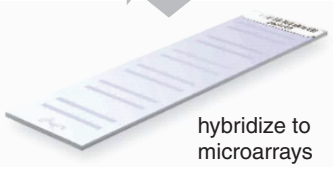

(c)

hsa-miR-10a-5p|\{hsa-miR-10a\}|21_43| hsa-miR-10a-5p|\{isomiR\}|22_44|

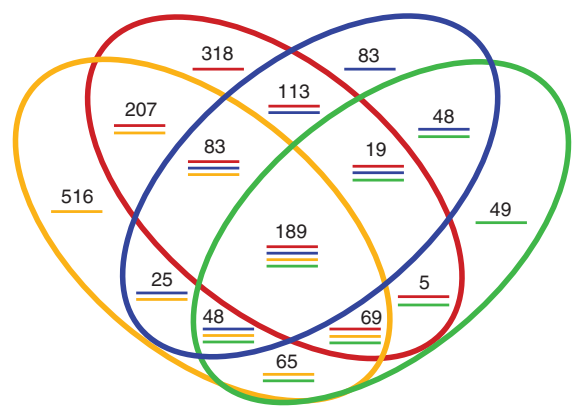

hsa-miR-10b-5p|\{hsa-miR-10b\}|26_48| hsa-miR-10b-5p|\{isomiR\}|27_49|

(e)

hsa-miR-10a-5p|\{hsa-miR-10a\}|21_43| hsa-miR-10a-5p|\{isomiR\}|22_44|

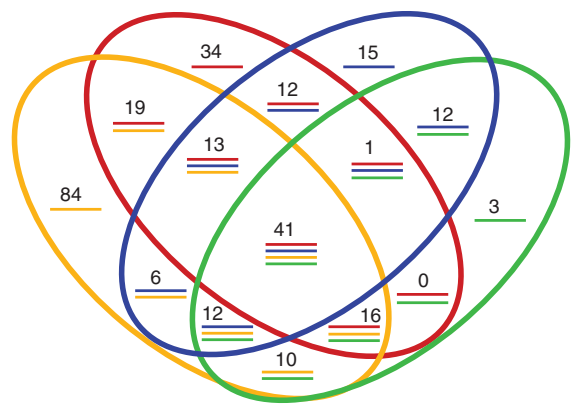

nsa-miR-10b-5p|\{hsa-miR-10b\}|26 48

hsa-miR-10b-5p|\{isomiR\}|27 49|

Figure 6 IsomiRs target similar molecules to their canonical miRNAs. (a) Schematic diagram depicting the laboratory workflow for the pulldown of mRNA targets of a synthetic biotinylated miRNA. (b) The miRNA expression profiles of the two miRNAs and corresponding shifted isomiRs selected for pull-down experiments. (c) A four-way Venn diagram depicting the overlap between the target genes detected by microarray analysis of pulled-down fractions. (d) The distribution of the number of miRNAs/isomiRs that target genes from significantly enriched pathways. There are more genes targeted by three or more isomiRs than would be expected by chance, and less genes targeted by two or fewer isomiRs than would be expected by chance. (e) A four-way Venn diagram displaying the specific targeting of the genes used in (d). 


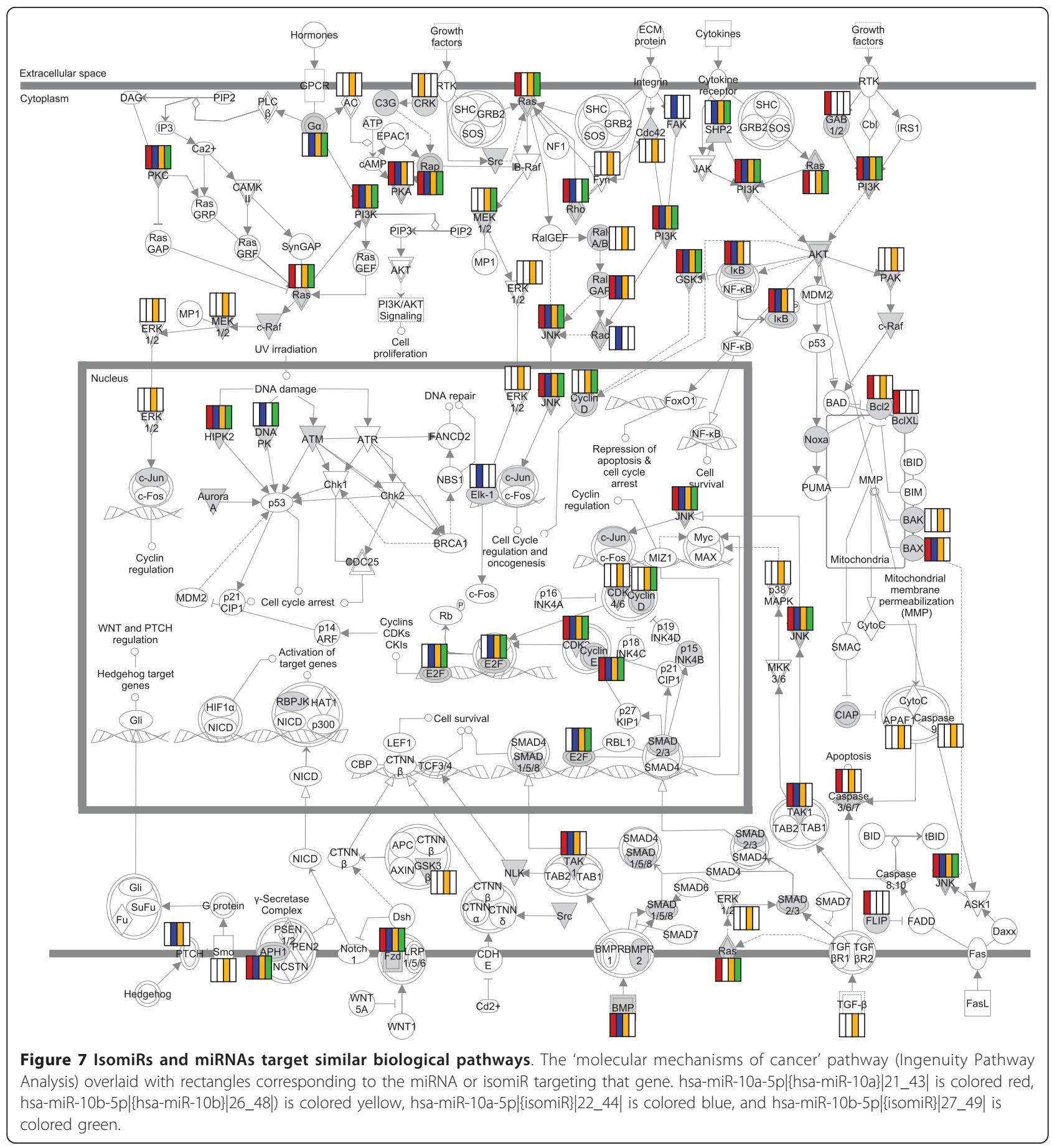

10 family (96 pathways; 284 genes). We then asked whether the distribution of miRNA targeting was different for this set of genes than would be expected by the distribution of genes that were pulled-down by any miRNA. We found a statistically significant difference between these two distributions (Chi-square value = 9.834; 3 degrees of freedom; $P=0.0200$ ), with an enrichment of genes that were targeted by three or more miRNAs/isomiRs (Figure 6d, e). These results confirm that genes targeted by multiple miRNAs/isomiRs are more likely to be enriched in a functional pathway, and that those that are targeted by less miRNAs/isomiRs are likely to be off-target interactions that are distant from the miRNA's central biology. When taken together, all these results support the hypothesis that miRNAs and their isomiRs act cooperatively to drive similar 
biology, and that the generation of isomiRs could increase both the signal to noise ratio and the potential dynamic range of miRNA effect (Figure 8).

\section{Discussion}

Almost every study analyzing miRNA-seq results has reported the presence of isomiRs, although their biological role has remained unclear. In this study, we have attempted to resolve the controversy by using thoroughly deep and extensively verified sequencing data and alignment strategies, operating under the assumption that more highly expressed sequences are likely to be more biologically relevant. Whilst we do not believe that biological importance correlates with transcriptional levels, we did aim to set conservative thresholds in order to counter the common argument that rare transcripts (particularly those with less than one transcript per cell) are less likely to be essential biological components. Arguably, this filtering may have been over-conservative, removing interesting and relevant biological models. For example, we did not detect 3' non-templated addition isomiRs above our stringent threshold, although this class of isomiR has been previously reported as developmentally regulated in Drosophila melanogaster [48].

Despite the aggressive filtering, most categories of isomiRs were detected well above the signal thresholds at comparable levels to canonical miRNA sequences, and their presence in individual donors across multiple tissues argues against these molecules being sequencing artifacts. Supporting the assertion that these molecules are functional, we find that isomiRs are specifically bound to translating mRNAs in the cytoplasm at similar proportions seen in total miRNA profiling (Figure 5e). This confirms that the association of isomiRs with AGO complexes is due to active inhibition of mRNA targets

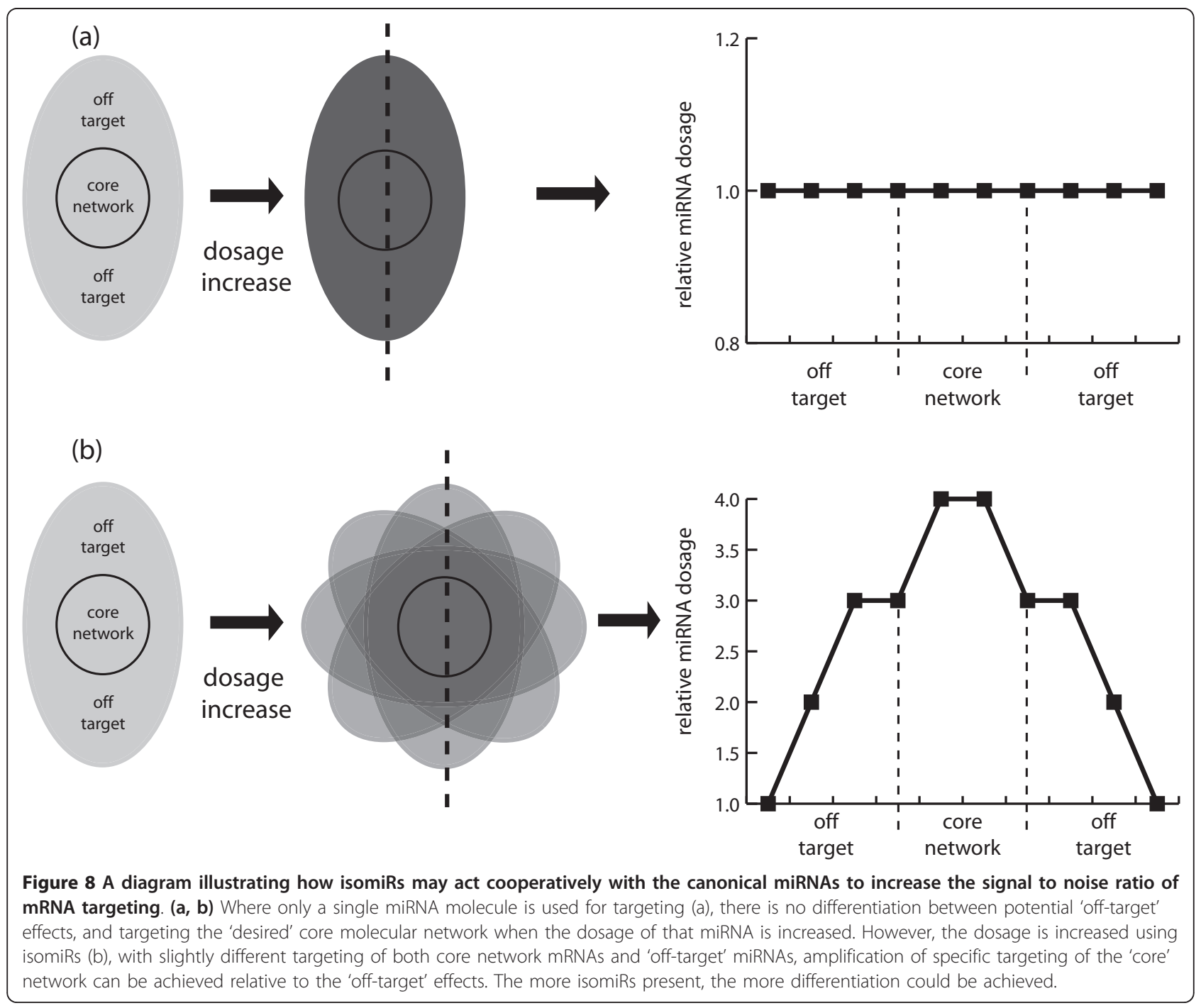


and is not the result of the accumulation of inactive complexes.

Some categories of isomiRs appear to be less relevant than others, and we find that isomiRs that differ only by nucleotide changes are more likely to be artifacts of the library preparation and/or sequencing based on their low absolute expression (Figure $3 \mathrm{~b}$ ), expression relative to their corresponding canonical miRNAs (Figure 3c), and their poor retention in active RISC (Table S7 in Additional file 1). However, these results cannot necessarily be generalized and should not be used to argue that every individual substitution isomiR is an artifact. Even within the substitution isomiRs, there are a few that pass both absolute and relative expression filters, and these could be candidate allelic-specific expression or RNA editing events. It is important to also consider the method of data generation when interpreting the results from sequencing: all enzymatic steps in library preparation include biases that manifest in the relative detection levels of miRNAs (and presumably isomiRs too) [52]. These biases could affect the detection of an individual isomiR relative to its canonical miRNA, or relative to other sequencing platforms, and could also distort the relative importance of isomiR classes. One such example is our finding that isomiR substitution and deletion events are less common than previously reported [51], a difference most likely due to the different sequencing technologies used. On a genome-wide scale the impact of such biases is often lessened, but not removed entirely. Further study to pinpoint the sources of bias in small RNA sequencing will allow the normalization of sequencing results, and a more accurate estimation of the relative abundance of isomiRs.

The depth of sequence used here has allowed us to study the number of miRNAs potentially undergoing alternative biogenesis as their primary method of production. This is an important consideration as almost half of the isomiRs detected in this study may have arisen as the result of ac-pre-miRNA cleavage and exonucleolytic activity. Support for this comes from significant ablation of expression from candidate acpre-miRNA hairpins when AGO2 is inactivated (Figure 4); however, cleavage imprecision from Drosha or Dicer may also account for this isomiR biogenesis. This explanation would require that the fidelity of cleavage at the $5^{\prime}$ end is greater than at the 3 ' end. For Dicer, this would seem to contradict the extensive structural evidence showing that the size of mature fragments is determined by the molecular distance between the PAZ and RNase III domains [69-72]. Imprecision in Drosha processing has been speculated to result in shifted-isomiRs [73], and while studies so far support a fixed-length cleavage of two helical turns [20], the experiments described here do not exclude imprecision as a contributing mechanism for isomiR biogenesis.

The use of the word 'imprecision' to describe isomiR production connotes images of waste, unnecessary byproducts, and non-functional output - but if this were true, why would such wasteful 'imprecision' be maintained through millions of years of evolution? In the case of miR-10 and other human hairpins, more isomiRs than canonical miRNAs are produced. Data from this study and others argue against isomiRs as non-functional by-products - they are associated with both AGO complexes $[45,54,55]$ and actively translating mRNAs (Figure 5). So what then is the biological function of these highly expressed isomiRs? The overlapping targeting (Figures 6c and 7) and high correlation of isomiRs (Figure S9 in Additional file 1) with their canonical miRNAs may answer one of the most intriguing paradoxes of miRNA function: the ability to drive specific biological phenotypes given the flexible nature of miRNAmRNA interactions. As few as six nucleotides are required in the 'seed' region to induce repression (and/ or degradation), and mismatches, bulges, and G:U wobble can all be tolerated if there is sufficient compensatory binding at the 3' end. Additionally, if there is a sequence match to the centered site, homology at the $5^{\prime}$ end of the miRNA is not required for function. The combination of these varied potential interactions leads directly to the prediction that miRNAs could target hundreds, if not thousands, of mRNAs, a prediction that has been confirmed by biotin pull-down assays in this study. Intriguingly, despite this apparent lack of specificity, miRNAs are involved in specific biological roles. Clues to how this specificity is achieved have been seen previously, with miRNAs targeting functionally related genetic networks either alone [64] or co-operatively with other miRNAs [74]. However, these genetic networks are small when compared to the hundreds of genuine mRNA targets of any given miRNA. Simply increasing the dosage of a single miRNA would not be expected to improve the 'on-target' to 'off-target' ratio; however, the addition of similar molecules with different repertoires of mRNA targets would distribute the 'off-target' hits while still targeting the core biological networks (Figure 8). Indeed, we did find a statistically significant enrichment for multiple miRNA targeting of the core miR-10 network; however, it is important to remember that we have profiled only one isomiR from each hairpin, where around 20 have been detected above the expression threshold. Not only would the isomiR-driven signal to noise ratio improve the sensitivity of the miRNA-controlled networks, but it could also increase exponentially the dynamic range of such targeting as combinatorial suppressive effects are exploited. An important caveat is that the hairpins studied here have more than $50 \%$ of 
their output as isomiRs. Such a model will probably not be applicable to hairpins where the proportion of isomiRs is low. However, there is scope for this model to be more widely applicable - when considering individual isomiRs, approximately $23 \%$ have expression the same as or greater than that of the canonical miRNA (Figure $3 c)$. Further study would be required to explore the extent that this model could contribute to gene regulation.

While the results shown here have emphasized what is similar between isomiR and canonical miRNA targeting, it is also possible that the differences could be biologically meaningful. An alternative (but not mutually exclusive) hypothesis is that isomiRs fine tune mRNA targeting to the requirements or responses of an individual cell type, and that cell-type-specific isomiR profiles may be invisible in our profiling of whole organs. This is not an easy hypothesis to test in the whole-organ profiling we have performed for this study, and is likely to require in-depth sequencing on individual cell types. At this stage, we are also unable to answer questions regarding the specific binding sites through which isomiRs target their mRNAs, and whether or not they competitively or cooperatively regulate their target mRNAs. Substantially more information about the wiring of these genetic networks could be gained by understanding exactly which miRNA binding sites are being occupied, something not currently possible with the microarray approach described here. Such attempts would be confounded by both the necessity to computationally infer potential binding sites, and the reliance on a reference genome to predict the presence of a binding site in the transcriptome of a cancer (and possibly highly mutated) cell line. Future studies should include simultaneous profiling of mRNA and miRNA populations across a number of tissues to determine the co-expression of miRNAs and their predicted targets, while systematic and comprehensive studies into individual isomiR targets and their precisely localized and experimentally confirmed binding sites will undoubtedly shed light on the hypotheses presented here.

\section{Conclusions}

IsomiRs have been reported in almost every sequencingbased study published to date, and yet their functional significance remains controversial. Our results demonstrate that isomiRs are functional, and that their biological role is likely to involve improving the signal to noise ratio in miRNA-mRNA targeting. These results have substantial implications for understanding the precise molecular mechanisms behind miRNA-controlled genetic networks, as the best target prediction programs are sensitive to a precise definition of a seed sequence, which can vary in isomiRs. However, incorporating knowledge of isomiR targets into systems biology approaches will greatly increase the sensitivity of miRNA-mRNA network discovery.

\section{Materials and methods}

\section{Library preparation and sequencing}

Construction of small RNA libraries was performed using both the Small RNA Expression Kit (SREK, Ambion, Austin, TX, USA), and the Whole Transcriptome Amplification Kit (WTAK, Ambion) according to the manufacturer's instructions. Briefly, a mixture of both 5' and 3' adaptors was combined with flashPAGE ${ }^{\mathrm{TM}}$ purified small RNAs (approximately 15 to 40 nucleotides) from FirstChoice ${ }^{\circledR}$ total RNAs (Ambion), and simultaneously ligated in a single reaction. cDNA was synthesized, and PCR amplified. During the PCR, sequencing adaptors were incorporated along with a unique six-nucleotide barcode sequence. Following library construction and purification, barcoded libraries were pooled and sequenced together using the Applied Biosystems (Beverly, MA, USA) SOLiD system.

These two protocols both use random hexamers attached to double-stranded adaptors to capture and position the small RNA for efficient ligation. SREK uses the 3' hexamer adaptor to prime cDNA synthesis, whereas WTAK uses a separate primer, and results in a different profile or errors in the 3' end of the miRNA. By examining the substitution isomiRs that are present in both libraries of the same tissue, we can exclude those that may be artifacts of library construction [SRA: SRP006043].

\section{Quantitative reverse-transcriptase PCR}

Triplicate reverse transcription reactions were done on total RNA samples using both the A and B Megaplex ${ }^{\mathrm{TM}}$ RT primer pools in conjunction with the TaqMan ${ }^{\circledR}$ MicroRNA Reverse Transcription Kit following the manufacturer's protocol. Reactions were run on an Applied Biosystems 7900HT. All arrays were analyzed using manual $\mathrm{C}_{\mathrm{T}}$ with automatic baseline adjustment and the threshold set at 0.1. A miRNA was considered to be detected by TaqMan if the average $C_{T}$ was $<35$.

\section{miRNA and isomiR analysis with miRNA-MATE}

Small RNA sequencing tags were aligned using miRNAMATE v1.1, an open source alignment tool designed in our laboratory specifically for color-space miRNA analysis. The pipeline is written in Perl, and source code, test data, and a comprehensive user manual are available to download from [75]. miRNA-MATE uses the recursive style of matching [50] for sensitive miRNA expression detection, but also can identify and strip the adaptor sequence to determine the precise ends of the captured miRNAs. Given the error rate of massive-scale 
sequencing (even when using the error-correcting properties of 2-base encoding by SOLiD) and the need to identify sequence-variant isomiRs, alignment against the entire genome would be impractical. Using vmatch [76], and allowing two nucleotide substitutions (no insertions or deletions), only 335 (30.5\%) human miRNAs could be uniquely placed against Hg19 (Table S2 in Additional file 1). Instead, we have restricted the search space to include only annotated pre-miRNA hairpin sequences (miRBase v15), and have allowed for up to two nucleotide substitutions during the alignment. The minimum length of tags matched was 20 nucleotides, as the specificity of matching drops dramatically at shorter lengths (data not shown). Tags were also matched using the same parameters to the complete repertoire of mature miRNA sequences from all species (miRBase v15) to determine the false positive matching rate for unrelated species. Sequences of rRNAs, tRNAs, and snoRNAs were downloaded from the UCSC Genome Browser [77], and aligned to as described above for miRNAs.

Wild-type and catalytically inactive AGO2 mutant miRNA-seq profiles [33] were downloaded from the NCBI Short Read Archive (accession number [SRP002411]). Fastq files were converted to color-space using a custom perl script, and mapped to $D$. rerio premiRNA hairpins (miRBase V17) using miRNA-MATE as described above. The two wild-type libraries (SRR042431 and SRR042433) were combined, but the depth of sequencing was still approximately ten times shallower than the mutant libraries. To correct for this massive sampling bias, we resampled the mutant library to produce a data set of the same depth as the wild-type library.

To correct for the distortion of proportional measures of expression (such as transcripts per million) that can happen when comparing very different expression profiles or libraries, we applied the trimmed mean of Mvalues scaling method [78]. All transcript per million (tpm) values reported in this manuscript have been corrected for these effective library sizes.

\section{Total, RNA-associated and polysome-associated miRNA preparation}

To prepare polysome-associated miRNAs, approximately $6 \times 10^{7} \mathrm{HeLa}$ cells were incubated with $10 \mu \mathrm{g} / \mathrm{ml}$ cycloheximide for 10 minutes at $37^{\circ} \mathrm{C}$, prior to lysis in hypotonic lysis buffer $\left(10 \mathrm{mM} \mathrm{KCl}, 1.5 \mathrm{mM} \mathrm{MgCl}_{2}, 10 \mathrm{mM}\right.$ Tris- $\mathrm{Cl}, \mathrm{pH} 7.4,10 \mu \mathrm{g} / \mathrm{ml}$ cycloheximide) and allowed to swell on ice for 10 minutes. Cells were homogenized using a 21 gauge needle, and centrifuged at 2,000 $\mathrm{g}$ for 2 minutes at $4{ }^{\circ} \mathrm{C}$ to pellet nuclei and unlysed cells. The lysate was brought to a total volume of $400 \mu \mathrm{l}$ with gradient buffer $(150 \mathrm{mM} \mathrm{KCl}, 5 \mathrm{mM} \mathrm{MgCl}$, $50 \mathrm{mM}$ Tris$\mathrm{Cl}, \mathrm{pH}$ 7.4). We prepared two gradients in Ultra-Clear
Thinwall $2.2 \mathrm{ml} 11 \times 34 \mathrm{~mm}$ centrifuge tubes (Beckman Instruments Inc., Fullerton, CA, USA), consisting of 1 $\mathrm{ml}$ of $1.5 \mathrm{M}$ sucrose in gradient buffer, then $1 \mathrm{ml}$ of 0.5 M sucrose in gradient buffer, and finally $200 \mu \mathrm{l}$ of cell lysate. The gradients were spun at $\geq 250,000 \mathrm{~g}$ for 5 hours at $4^{\circ} \mathrm{C}$. Fractions $(60 \mu \mathrm{l})$ were removed from the top of the gradient, and the UV absorbance was measured at $260 \mathrm{~nm}$. Fractions containing polysomes were pooled, and RNA was extracted using an RNeasy kit (Qiagen, Doncaster, VIC, Australia) according to the manufacturer's instructions. miRNAs were eluted from the mRNAs by heat $\left(95^{\circ} \mathrm{C}\right.$ for 2 minutes $)$, and purified from the large RNAs using flashPAGE Fractionator and the flashPAGE Reaction Clean-up Kit (Ambion) according to the manufacturer's instructions.

RNA-associated miRNAs were collected by purifying total RNA from HeLa cells using an RNeasy column (Qiagen) according to the manufacturer's instructions. miRNAs were eluted as for polysome-associated miRNAs. Total HeLa miRNAs were purified using a miRNeasy column (Qiagen) according to the manufacturer's instructions. All miRNAs were then captured and sequenced as described above.

To confirm that miRNAs were not retained on the RNeasy columns in the absence of long RNAs, total RNA processed with both miRNeasy and RNeasy columns were heat denatured for 2 minutes at $75^{\circ} \mathrm{C}$, and re-purified through a new miRNeasy or RNeasy column, respectively. RNeasy purified RNA was also re-purified through a new RNeasy column without heat denaturation. cDNA was prepared using a TaqMan MicroRNA Reverse Transcription Kit (Life Technologies, Foster City, CA, USA). qRT-PCR was performed using SYBR green PCR master-mix (Life Technologies) on an ABI 7900 HT 7 Real time PCR system. Expression values were normalized to RNU6B.

\section{Biotinylated miRNA pull-downs}

Synthetic biotin-labeled miRNA duplexes (200 pmoles; Table S8 in Additional file 1; Integrated DNA Technologies San Diego, CA, USA) were transfected into $4 \times 10^{6}$ HEK293T cells using HiPerFect Transfection Reagent (Qiagen). Cells were harvested after 24 hours, and lysed in hypotonic lysis buffer $\left(10 \mathrm{mM} \mathrm{KCl}, 1.5 \mathrm{mM} \mathrm{MgCl}_{2}\right.$, $10 \mathrm{mM}$ Tris-Cl pH 7.5, 5 mM DTT, 0.5\% NP-40, $60 \mathrm{U} /$ ML SUPERase $\cdot$ In (Ambion) and $1 \times$ Complete Mini protease inhibitor (Roche, Dee Why, NSW, Australia). Cell debris was cleared by centrifugation $\left(\geq 10,000 \mathrm{~g}\right.$ at $4^{\circ} \mathrm{C}$ for 2 minutes). The supernatant was transferred to a clean tube, and $\mathrm{NaCl}$ was added to a final concentration of $1 \mathrm{M}$. myOne C1 Dynabeads (25 $\mu \mathrm{l}$; Invitrogen, Thornton, NSW, Australia) were pre-blocked with $1 \mu \mathrm{g}$ / $\mu \mathrm{l}$ bovine serum albumin and $1 \mu \mathrm{g} / \mu \mathrm{l}$ yeast tRNA (Invitrogen), and incubated with the supernatant for 30 
minutes at room temperature. Beads were then washed with hypotonic lysis buffer and $1 \mathrm{M} \mathrm{NaCl}$ before RNA extraction using an RNeasy Kit (Qiagen) according to the manufacturer's instructions.

\section{Microarray hybridizations and analysis}

MicroRNA microarray samples were prepared using the Agilent miRNA Microarray System protocol. An input of $100 \mathrm{ng}$ total RNA was labeled and hybridized to the Agilent Human miRNA array (AMADID 016436) for 20 hours at $55^{\circ} \mathrm{C}$. The slides were scanned using the Agilent Scanner G2505B US22502591 (Agilent Technologies) and data were extracted using the Feature Extraction software v9.5.3.1 (Agilent Technologies).

Fifty nanograms of the mRNA samples captured in the biotin pulldowns were amplified and labeled using the Illumina TotalPrep RNA Amplification Kit as per the manufacturer's instructions. Post-amplification RNA quality control was performed using a NanoDrop (Thermo Scientific, Bellefonte, PA, USA) and an Agilent 2100 Bioanalyzer. Amplified RNA (750 ng) was hybridized onto Illumina human HT-12 chips (V3 and V4), and scanned on a Bead Array Reader (Illumina) [GEO: GSE29101]. Expression measurements were extracted using the GenomeStudio (version 2009.1; Illumina) software. The data from all microarrays were first normalized using the median percentile rank approach [77]. Thresholds of 0.76 and 0.95 were selected for miR-17$5 \mathrm{p}$ and the miR-10 family, respectively, and genes that had that rank or higher were selected for GSEA. GSEA was performed using Ingenuity Pathway Analysis [79].

\section{Accession numbers}

The sequencing data reported in this study can be obtained from the NCBI Short Read Archive (SRA) under accession number [SRP006043], and the microarray data are available from the Gene Expression Omnibus (GEO) under accession number [GSE29101].

\section{Additional material}

Additional file 1: Supplementary figures, figure legends and tables

Additional file 2: miRNA-MATE source code and user manual.

Additional file 3: A description of the proposed nomenclature for isomiRs.

Additional file 4: Tab-delimited text file of the raw counts of isomers detected in the libraries created in this study.

\footnotetext{
Abbreviations

AGO2: argonaut 2; ac-pre-miRNA, argonaut cleaved precursor miRNA hairpin; GSEA: gene set enrichment analysis; miRNA: microRNA; miRNA-seq: microRNA sequencing; NCBI: National Center for Biotechnology Information; pre-miRNA: precursor microRNA hairpin; qRT-PCR: quantitative reversetranscriptase polymerase chain reaction; RISC: RNA induced silencing
}

complex; RNA-seq: RNA sequencing; snoRNA: small nucleolar RNA; SREK: Small RNA Expression Kit; tpm: transcripts per million; WTAK: Whole Transcriptome Amplification Kit.

\section{Acknowledgements}

This work was supported by Australian Research Council (ARC) Discovery Project Grants DP0988754 and DP1093164. NC is supported by an ARC Postdoctoral Fellowship, and SMG is supported by an Australian National Health and Medical Research Council (NHMRC) Senior Research Fellowship. We are grateful for the helpful discussions with all members of QCMG, and we are particularly thankful to JV Pearson, DF Taylor, and S Wood for HPC infrastructure and support. We also thank Ryan Taft, Selene Fernandez Valverde, and John Mattick for helpful discussions and advice.

\section{Author details}

${ }^{1}$ Queensland Centre for Medical Genomics, Institute for Molecular Bioscience, The University of Queensland, St Lucia, Queensland, 4072, Australia. ${ }^{2}$ Life Technologies, 2130 Woodward St, Austin, TX 78744, USA. ${ }^{3}$ Life Technologies, 800 Lincoln Centre Dr., Foster City, CA 94404, USA. ${ }^{4}$ Life Technologies, 500 Cummings Center, Suite 2400, Beverly, MA 01915, USA. ${ }^{5}$ Epicentre (An Illumina Company), 726 Post Rd, Madison, WI 53713, USA.

\section{Authors' contributions}

NC conceived and designed the experiments, performed the experiments, analyzed the data and wrote the manuscript. SK and SMG conceived and designed the experiments and wrote the manuscript. SW, JG, KL, SH, ALS, $\mathrm{EN}, \mathrm{KK}, \mathrm{BBG}$, and KN performed the experiments. QX, CB, HCM, XW, JAS, NM, DLW, KSK, NW, and JS analyzed the data. CL, Jl, KM, and KB contributed reagents, materials and/or analysis tools. All authors have read and approved the manuscript for publication.

\section{Competing interests}

$J G, K L, S H, C B, X W, C L, J I, K M, K B$, and $S K$ are currently or were previously employed by Life Technologies, a manufacturer of sequencing instrumentation and reagents. No other authors have any competing interests.

Received: 12 July 2011 Revised: 21 September 2011

Accepted: 30 December 2011 Published: 30 December 2011

\section{References}

1. Humphreys DT, Westman BJ, Martin DIK, Preiss T: MicroRNAs control translation initiation by inhibiting eukaryotic initiation factor 4E/cap and poly(A) tail function. Proc Natl Acad Sci USA 2005, 102:16961-16966.

2. Maroney Pa, Yu Y, Fisher J, Nilsen TW: Evidence that microRNAs are associated with translating messenger RNAs in human cells. Nat Struct Mol Biol 2006, 13:1102-1107.

3. Mathonnet G, Fabian MR, Svitkin $\mathrm{V}$, Parsyan A, Huck L, Murata T, Biffo S, Merrick WC, Darzynkiewicz E, Pillai RS, Filipowicz W, Duchaine TF, Sonenberg N: MicroRNA inhibition of translation initiation in vitro by targeting the cap-binding complex elF4F. Science 2007, 317:1764-1767.

4. Nottrott S, Simard MJ, Richter JD: Human let-7a miRNA blocks protein production on actively translating polyribosomes. Nat Struct Mol Biol 2006, 13:1108-1114.

5. Petersen CP, Bordeleau M-E, Pelletier J, Sharp P: Short RNAs repress translation after initiation in mammalian cells. Mol Cell 2006, 21:533-542.

6. Pillai RS, Bhattacharyya SN, Artus CG, Zoller T, Cougot N, Basyuk E, Bertrand E, Filipowicz W: Inhibition of translational initiation by Let-7 MicroRNA in human cells. Science 2005, 309:1573-1576.

7. Liu J, Valencia-Sanchez MA, Hannon GJ, Parker R: MicroRNA-dependent localization of targeted mRNAs to mammalian P-bodies. Nat Cell Biol 2005, 7:719-723.

8. Giraldez AJ, Mishima Y, Rihel J, Grocock RJ, Van Dongen S, Inoue K, Enright AJ, Schier AF: Zebrafish MiR-430 promotes deadenylation and clearance of maternal mRNAs. Science 2006, 312:75-79.

9. Valencia-Sanchez MA, Liu J, Hannon GJ, Parker R: Control of translation and mRNA degradation by miRNAs and siRNAs. Genes Dev 2006, 20:515-524.

10. Wu L, Fan J, Belasco JG: MicroRNAs direct rapid deadenylation of mRNA. Proc Natl Acad Sci USA 2006, 103:4034-4039. 
11. Hornstein E, Mansfield JH, Yekta S, Hu JK-H, Harfe BD, McManus MT, Baskerville S, Bartel DP, Tabin CJ: The microRNA miR-196 acts upstream of Hoxb8 and Shh in limb development. Nature 2005, 438:671-674.

12. Chen C-Z, Li L, Lodish HF, Bartel DP: MicroRNAs modulate hematopoietic lineage differentiation. Science 2004, 303:83-86.

13. Giraldez AJ, Cinalli RM, Glasner ME, Enright AJ, Thomson JM, Baskerville S, Hammond SM, Bartel DP, Schier AF: MicroRNAs regulate brain morphogenesis in zebrafish. Science 2005, 308:833-838.

14. Zhao Y, Samal E, Srivastava D: Serum response factor regulates a musclespecific microRNA that targets Hand2 during cardiogenesis. Nature 2005, 436:214-220.

15. He L, Thomson JM, Hemann MT, Hernando-Monge E, Mu D, Goodson S, Powers S, Cordon-Cardo C, Lowe SW, Hannon GJ, Hammond SM: A microRNA polycistron as a potential human oncogene. Nature 2005, 435:828-833

16. Chen $X$, Guo $X$, Zhang H, Xiang Y, Chen J, Yin Y, Cai X, Wang K, Wang G, Ba Y, Zhu L, Wang J, Yang R, Zhang Y, Ren Z, Zen K, Zhang J, Zhang C-Y: Role of miR-143 targeting KRAS in colorectal tumorigenesis. Oncogene 2009, 28:1385-1392.

17. Tavazoie SF, Alarcón C, Oskarsson T, Padua D, Wang Q, Bos PD, Gerald WL, Massagué J: Endogenous human microRNAs that suppress breast cancer metastasis. Nature 2008, 451:147-152

18. Krol J, Loedige I, Filipowicz W: The widespread regulation of microRNA biogenesis, function and decay. Nat Rev Genet 2010, 11:597-610.

19. Han J, Lee Y, Yeom K-H, Kim Y-K, Jin H, Kim VN: The Drosha-DGCR8 complex in primary microRNA processing. Genes Dev 2004, 18:3016-3027.

20. Han J, Lee Y, Yeom K-H, Nam J-W, Heo I, Rhee J-K, Sohn SY, Cho Y, Zhang B-T, Kim VN: Molecular basis for the recognition of primary microRNAs by the Drosha-DGCR8 complex. Cell 2006, 125:887-901.

21. Okamura K, Hagen JW, Duan H, Tyler DM, Lai EC: The mirtron pathway generates microRNA-class regulatory RNAs in Drosophila. Cell 2007, 130:89-100.

22. Ruby JG, Jan CH, Bartel DP: Intronic microRNA precursors that bypass Drosha processing. Nature 2007, 448:83-86.

23. Yi R, Qin Y, Macara IG, Cullen BR: Exportin-5 mediates the nuclear export of pre-microRNAs and short hairpin RNAs. Genes Dev 2003, 17:3011-3016.

24. Gregory Rl, Chendrimada TP, Cooch N, Shiekhattar R: Human RISC couples microRNA biogenesis and posttranscriptional gene silencing. Cell 2005, 123:631-640

25. Hutvágner G, McLachlan J, Pasquinelli aE, Bálint E, Tuschl T, Zamore PD: A cellular function for the RNA-interference enzyme Dicer in the maturation of the let-7 small temporal RNA. Science 2001, 293:834-838,

26. Khvorova A, Reynolds A, Jayasena SD: Functional siRNAs and miRNAs exhibit strand bias. Cell 2003, 115:209-216.

27. Schwarz DS, Hutvágner G, Du T, Xu Z, Aronin N, Zamore PD: Asymmetry in the assembly of the RNAi enzyme complex. Cell 2003, 115:199-208.

28. Czech B, Zhou R, Erlich Y, Brennecke J, Binari R, Villalta C, Gordon A, Perrimon N, Hannon GJ: Hierarchical rules for Argonaute loading in Drosophila. Mol Cell 2009, 36:445-456.

29. Ghildiyal M, Xu J, Seitz H, Weng Z, Zamore PD: Sorting of Drosophila small silencing RNAs partitions microRNA* strands into the RNA interference pathway. RNA 2010, 16:43-56

30. Okamura K, Liu N, Lai EC: Distinct mechanisms for microRNA strand selection by Drosophila Argonautes. Mol Cell 2009, 36:431-444.

31. Diederichs $S$, Haber Da: Dual role for argonautes in microRNA processing and posttranscriptional regulation of microRNA expression. Cell 2007, 131:1097-1108.

32. Cheloufi S, Santos CO Dos, Chong MMW, Hannon GJ: A dicerindependent miRNA biogenesis pathway that requires Ago catalysis. Nature 2010, 465:584-589.

33. Cifuentes D, Xue H, Taylor DW, Patnode H, Mishima Y, Cheloufi S, Ma E, Mane S, Hannon GJ, Lawson N, Wolfe S, Giraldez AJ: A novel miRNA processing pathway independent of Dicer requires Argonaute2 catalytic activity. Science 2010, 328:1694-1698.

34. Mortazavi A, Williams BA, McCue K, Schaeffer L, Wold B: Mapping and quantifying mammalian transcriptomes by RNA-Seq. Nat Methods 2008, 5:621-628.

35. Cloonan N, Forrest ARR, Kolle G, Gardiner BBA, Faulkner GJ, Brown MK, Taylor DF, Steptoe AL, Wani S, Bethel G, Robertson AJ, Perkins AC, Bruce SJ, Lee CC, Ranade SS, Peckham HE, Manning JM, McKernan KJ, Grimmond SM:
Stem cell transcriptome profiling via massive-scale mRNA sequencing. Nat Methods 2008, 5:613-619.

36. Cloonan N, Grimmond SM: Transcriptome content and dynamics at single-nucleotide resolution. Genome Biol 2008, 9:234.

37. Morin RD, O'Connor MD, Griffith M, Kuchenbauer F, Delaney A, Prabhu A-L, Zhao Y, McDonald H, Zeng T, Hirst M, Eaves CJ, Marra M: Application of massively parallel sequencing to microRNA profiling and discovery in human embryonic stem cells. Genome Res 2008, 18:610-621.

38. Kim J, Cho IS, Hong JS, Choi YK, Kim H, Lee YS: Identification and characterization of new microRNAs from pig. Mamm Genome 2008, 19:570-580.

39. Reese Ta, Xia J, Johnson LS, Zhou X, Zhang W, Virgin HW: Identification of novel microRNA-like molecules generated from herpesvirus and host tRNA transcripts. J Virol 2010, 84:10344-10353.

40. de Hoon MJ, Taft RJ, Hashimoto T, Kanamori-Katayama M, Kawaji H, Kawano M, Kishima M, Lassmann T, Faulkner GJ, Mattick JS, Daub CO, Carninci P, Kawai J, Suzuki H, Hayashizaki Y: Cross-mapping and the identification of editing sites in mature microRNAs in high-throughput sequencing libraries. Genome Res 2010, 20:257-264.

41. Sdassi N, Silveri L, Laubier J, Tilly G, Costa J, Layani S, Vilotte J-L, Le Provost F: Identification and characterization of new miRNAs cloned from normal mouse mammary gland. BMC Genomics 2009, 10:149.

42. Ruby JG, Stark A, Johnston WK, Kellis M, Bartel DP, Lai EC: Evolution, biogenesis, expression, and target predictions of a substantially expanded set of Drosophila microRNAs. Genome Res 2007, 17:1850-1864.

43. Ruby JG, Jan C, Player C, Axtell MJ, Lee W, Nusbaum C, Ge H, Bartel DP: Large-scale sequencing reveals 21U-RNAs and additional microRNAs and endogenous siRNAs in C. elegans. Cell 2006, 127:1193-1207.

44. Cummins JM, He Y, Leary RJ, Pagliarini R, Diaz La, Sjoblom T, Barad O, Bentwich Z, Szafranska AE, Labourier E, Raymond CK, Roberts BS, Juhl H, Kinzler KW, Vogelstein B, Velculescu VE: The colorectal microRNAome. Proc Natl Acad Sci USA 2006, 103:3687-3692.

45. Ebhardt HA, Tsang HH, Dai DC, Liu Y, Bostan B, Fahlman RP: Meta-analysis of small RNA-sequencing errors reveals ubiquitous post-transcriptional RNA modifications. Nucleic Acids Res 2009, 37:2461-2470.

46. Pantano L, Estivill $X$, Martí E: SeqBuster, a bioinformatic tool for the processing and analysis of small RNAs datasets, reveals ubiquitous miRNA modifications in human embryonic cells. Nucleic Acids Res 2010, 38:e34.

47. Lee LW, Zhang S, Etheridge A, Ma L, Martin D, Galas D, Wang K: Complexity of the microRNA repertoire revealed by next generation sequencing. Rna 2010, 16:2170-2180.

48. Fernandez-Valverde SL, Taft RJ, Mattick JS: Dynamic isomiR regulation in Drosophila development. Rna 2010, 16:1881-1888.

49. Kozomara A, Griffiths-Jones S: miRBase: integrating microRNA annotation and deep-sequencing data. Nucleic Acids Res 2011, 39:D152-157.

50. Cloonan N, Xu Q, Faulkner GJ, Taylor DF, Tang DTP, Kolle G, Grimmond SM: RNA-MATE: a recursive mapping strategy for high-throughput RNAsequencing data. Bioinformatics 2009, 25:2615-2616.

51. Reid JG, Nagaraja AK, Lynn FC, Drabek RB, Muzny DM, Shaw Ca, Weiss MK, Naghavi AO, Khan M, Zhu H, Tennakoon J, Gunaratne GH, Corry DB, Miller J, McManus MT, German MS, Gibbs Ra, Matzuk MM, Gunaratne PH: Mouse let-7 miRNA populations exhibit RNA editing that is constrained in the $5^{\prime}$-seed/cleavage/anchor regions and stabilize predicted mmu-let-7a: mRNA duplexes. Genome Res 2008, 18:1571-1581.

52. Linsen SEV, de Wit E, Janssens G, Heater S, Chapman L, Parkin RK, Fritz B, Wyman SK, de Bruijn E, Voest EE, Kuersten S, Tewari M, Cuppen E: Limitations and possibilities of small RNA digital gene expression profiling. Nat Methods 2009, 6:474-476.

53. He F, Li X, Spatrick P, Casillo R, Dong S, Jacobson A: Genome-wide analysis of mRNAs regulated by the nonsense-mediated and $5^{\prime}$ to $3^{\prime}$ mRNA decay pathways in yeast. Mol Cell 2003, 12:1439-1452.

54. Martí E, Pantano L, Bañez-Coronel M, Llorens F, Miñones-Moyano E, Porta S, Sumoy L, Ferrer I, Estivill X: A myriad of miRNA variants in control and Huntington's disease brain regions detected by massively parallel sequencing. Nucleic Acids Res 2010, 38:1-17.

55. Siomi MC, Siomi H: Characterization of endogenous human Argonautes and their miRNA partners in RNA silencing. Nucleic Acids SympSer (Oxf) 2008, 105:59-60. 
56. Maroney PA, Yu Y, Fisher J, Nilsen TW: Evidence that microRNAs are associated with translating messenger RNAs in human cells. Nat Struct Mol Biol 2006, 13:1102-1107.

57. Fukagawa T, Nogami M, Yoshikawa M, Ikeno M, Okazaki T, Takami Y, Nakayama T, Oshimura M: Dicer is essential for formation of the heterochromatin structure in vertebrate cells. Nat Cell Biol 2004 6:784-791.

58. Bartel DP: MicroRNAs: target recognition and regulatory functions. Cell 2009, 136:215-233.

59. Lewis BP, hung IShih, Jones-Rhoades MW, Bartel DP, Burge CB: Prediction of mammalian microRNA targets. Cell 2003, 115:787-798.

60. Krek A, Grün D, Poy MN, Wolf R, Rosenberg L, Epstein EJ, MacMenamin P, da Piedade I, Gunsalus KC, Stoffel M, Rajewsky N: Combinatorial microRNA target predictions. Nat Genet 2005, 37:495-500.

61. Lewis BP, Burge CB, Bartel DP: Conserved seed pairing, often flanked by adenosines, indicates that thousands of human genes are microRNA targets. Cell 2005, 120:15-20.

62. Orom UA, Lund AH: Isolation of microRNA targets using biotinylated synthetic microRNAs. Methods 2007, 43:162-165.

63. Ørom UA, Nielsen FC, Lund AH: MicroRNA-10a binds the $5^{\prime}$ UTR of ribosomal protein mRNAs and enhances their translation. Mol Cell 2008, 30:460-471.

64. Cloonan N, Brown MK, Steptoe AL, Wani S, Chan WL, Forrest ARR, Kolle G, Gabrielli B, Grimmond SM: The miR-17-5p microRNA is a key regulator of the G1/S phase cell cycle transition. Genome Biol 2008, 9:R127.

65. Brock M, Trenkmann M, Gay RE, Michel Ba, Gay S, Fischler M, Ulrich S, Speich R, Huber LC: Interleukin-6 modulates the expression of the bone morphogenic protein receptor type II through a novel STAT3-microRNA cluster 17/92 pathway. Circ Res 2009, 104:1184-1191.

66. Dews M, Fox JL, Hultine S, Sundaram P, Wang W, Liu YY, Furth E, Enders GH, El-Deiry W, Schelter JM, Cleary Ma, Thomas-Tikhonenko A: The myc-miR-17 92 axis blunts TGF\{beta\} signaling and production of multiple TGF\{beta\}-dependent antiangiogenic factors. Cancer Res 2010, 70:8233-8246.

67. Hossain A, Kuo MT, Saunders GF: Mir-17-5p regulates breast cancer cell proliferation by inhibiting translation of AIB1 mRNA. Mol Cell Biol 2006, 26:8191-8201.

68. Lund aH: miR-10 in development and cancer. Cell Death Differ 2010, 17:209-214.

69. Ma J-B, Ye K, Patel DJ: Structural basis for overhang-specific small interfering RNA recognition by the PAZ domain. Nature 2004, 429:318-322.

70. Lingel A, Simon B, Izaurralde E, Sattler M: Nucleic acid 3'-end recognition by the Argonaute2 PAZ domain. Nat Struct Mol Biol 2004, 11:576-577.

71. Zhang H, Kolb Fa, Brondani V, Billy E, Filipowicz W: Human Dicer preferentially cleaves dsRNAs at their termini without a requirement for ATP. EMBO J 2002, 21:5875-5885.

72. Macrae IJ, Zhou K, Li F, Repic A, Brooks AN, Cande WZ, Adams PD, Doudna J: Structural basis for double-stranded RNA processing by Dicer. Science 2006, 311:195-198.

73. Wu H, Ye C, Ramirez D, Manjunath N: Alternative processing of primary microRNA transcripts by Drosha generates $5^{\prime}$ end variation of mature microRNA. Plos One 2009, 4:e7566.

74. Forrest aRR, Kanamori-Katayama M, Tomaru Y, Lassmann T, Ninomiya N, Takahashi Y, de Hoon MJL, Kubosaki A, Kaiho A, Suzuki M, Yasuda J, Kawai J, Hayashizaki Y, Hume D, Suzuki H: Induction of microRNAs, mir155, mir-222, mir-424 and mir-503, promotes monocytic differentiation through combinatorial regulation. Leukemia 2010, 24:460-466.

75. Supplementary material.. [http://grimmond.imb.uq.edu.au/miRNA-MATE/].

76. The Vmatch Large Scale Sequence Analysis Software.. [http://www. vmatch.de/l.

77. Kuhn RM, Karolchik D, Zweig AS, Wang T, Smith KE, Rosenbloom KR, Rhead B, Raney BJ, Pohl A, Pheasant M, Meyer L, Hsu F, Hinrichs AS, Harte RA, Giardine B, Fujita P, Diekhans M, Dreszer T, Clawson H, Barber GP, Haussler D, Kent WJ: The UCSC Genome Browser Database: update 2009. Nucleic Acids Res 2009, 37:D755-761

78. Robinson MD, Oshlack A: A scaling normalization method for differential expression analysis of RNA-seq data. Genome Biol 2010, 11:R25.

79. Ingenuity Systems.. [http://www.ingenuity.com]. doi:10.1186/gb-2011-12-12-r126

Cite this article as: Cloonan et al: MicroRNAs and their isomiRs function cooperatively to target common biological pathways. Genome Biology 2011 12:R126.

\section{Submit your next manuscript to BioMed Central and take full advantage of:}

- Convenient online submission

- Thorough peer review

- No space constraints or color figure charges

- Immediate publication on acceptance

- Inclusion in PubMed, CAS, Scopus and Google Scholar

- Research which is freely available for redistribution

Submit your manuscript at www.biomedcentral.com/submit
Ciomed Central 